\title{
Differential Analysis of Serum Principal Components Treated with Compound Sophora Decoction and Related Compounds Based on High-Resolution Mass Spectrometry (HRMS)
}

\author{
Wanjin Sun, ${ }^{1,2}$ Junjie Zhang, ${ }^{3,4}$ Conghui Zhou, ${ }^{1,2}$ Bin Yan, ${ }^{1,2}$ Quan Cai, ${ }^{4}$ Hongxia He, \\ Xueyun Duan $\left(\mathbb{1},,^{1,2}\right.$ and Heng Fan $\mathbb{1}^{4}$ \\ ${ }^{1}$ Department of Pharmacy, Hubei Provincial Hospital of Traditional Chinese Medicine, Wuhan 430061, China \\ ${ }^{2}$ Department of Pharmacy, Hubei Province Academy of Traditional Chinese Medicine, Wuhan 430074, China \\ ${ }^{3}$ Graduate School, Tianjin University of Traditional Chinese Medicine, Tianjin 300000, China \\ ${ }^{4}$ Department of Integrated Traditional Chinese and Western Medicine, Union Hospital, Tongji Medical College, \\ Huazhong University of Science and Technology, Wuhan 430022, China
}

Correspondence should be addressed to Xueyun Duan; 1046625326@qq.com and Heng Fan; fanheng009@aliyun.com

Received 26 May 2020; Revised 11 August 2020; Accepted 16 September 2020; Published 30 September 2020

Academic Editor: Jairo Kennup Bastos

Copyright (C) 2020 Wanjin Sun et al. This is an open access article distributed under the Creative Commons Attribution License, which permits unrestricted use, distribution, and reproduction in any medium, provided the original work is properly cited.

Objective. To compare the differences in the serum principal components in ulcerative colitis- (UC-) induced rats, treated with compound Sophora decoction, matrine, oxymatrine monomer mixture, and indirubin monomer, and to provide a modern scientific basis for elucidating the clinical efficacy of compound Sophora decoction for the treatment of UC. Methods. The serum samples of rats from each group were obtained after drug administration, and the serum principal components of each group were analyzed by high-resolution mass spectrometry. Agilent Eclipse XDB C18 chromatographic column $(100 \mathrm{~mm} \times 2.1 \mathrm{~mm}, 3.5 \mathrm{~m})$ was used for separation. The mobile phase was water (A) and methanol (B) (0.1\% formic acid) gradient elution, $0-3 \mathrm{~min}(\mathrm{~B}: 20 \%-$ 40\%), 3-10 $\min$ (B: 40\%-54\%), 10-25 $\min$ (B: 54\%), 25-35 min (B: 54\%-70\%), 35-45 min (B: 70\%-80\%), 45-50 min (B: 80\%), 50-60 $\min$ (B: $80 \%-100 \%$ ), 70-72 $\min$ (B: 100\%-20\%), and 72-77 $\min$ (B: $20 \%$ ); flow rate, $300 \mu \mathrm{L} / \mathrm{min}$; column temperature, $40^{\circ} \mathrm{C}$; and injection volume, $10 \mu \mathrm{L}$. ESI source was selected and scanned in the positive and negative ion modes. The scanning range was 70-1500 m/z; ion-source gas 1 (GS1): 55 psi; ion-source gas 2 (GS2): 60 psi; CUR: 30 psi; ion-source temperature (TEM): $550^{\circ} \mathrm{C}$; ion-source voltage (ISVF) : $5500 \mathrm{~V} /-4500 \mathrm{~V}$; decluster voltage (DP): $100 \mathrm{~V}$; collision energy (CE): $35 \mathrm{~V} /-35 \mathrm{~V}$; collision energy gain (CES) : $15 \mathrm{~V} /-15 \mathrm{~V}$; and data acquisition mode: IDA. After the data from each group were imported into MarkView 1.3, the molecular weights and retention times of different substances were obtained and qualitatively analyzed by ChemSpider and PeakView 2.0. Results. In the negative ion mode, 26 differential compounds were identified in the compound Sophora decoction group (FFKST) compared to the model group (M), and 18 differential compounds were identified in the matrine and oxymatrine group (KST) compared to the model group (M). In the positive ion mode, 11 and 7 differential compounds were identified in the compound Sophora decoction group (FFKST) and the matrine and oxymatrine group (KST) compared to the model group (M), respectively. The responses of all compounds in each group were compared with each other. As the different principal component substances in the indirubin group (DYH) displayed little correlation with other groups, the different components in this group were not researched thoroughly. Conclusion. By comparing the differences in the serum principal components from each administration group, we found that the FFKST group exhibited enhanced synthesis of the serum principal components; however, the compound doses of matrine and oxymatrine monomers did not exhibit the same changes in the serum principal components of UC-induced rats. Finally, the traditional Chinese medicine compound is more advantageous than monomers. 


\section{Introduction}

Ulcerative colitis (UC) is a recurrent inflammatory bowel disease, characterized by chronic inflammation and ulcer formation in the colonic mucosa. The disease manifests pus and blood. The lesions are located mostly in the sigmoid colon and rectum, but they can spread to the descending colon, even affecting the entire colon. The disease is longlasting and recurrent $[1,2]$. The compound Sophora decoction is one of the Chinese herbal medicines, which has exhibited remarkable curative effects in the clinical treatment of UC. It consists of Sophora flavescens Ait., Sanguisorba officinalis L., Indigo naturalis, Bletilla striata Diels., and Glycyrrhiza uralensis Fisch. In the preliminary results, matrine and oxymatrine displayed significant anti-inflammatory activities. They reduced intestinal mucosal inflammation in experimentally induced colitis by regulating NOD $2 / N F-\kappa \mathrm{B}$ and $\beta 2 \mathrm{AR}-\beta$-arrestin- $2-\mathrm{NF}-\kappa \mathrm{B}$ signal transduction pathways (important components to evaluate the polyjuice potion) $[3,4]$. Simultaneously, indirubin promoted the expression of claudin-1, occludin, zo-1, and jam1 , suggesting that indirubin is the main effective component for UC treatment [5]. Chinese traditional medicine functions in a "multicomponent and multitarget" pathway for disease treatment by the synergistic action of its components $[6,7]$. The effect of a compound or monomer depends upon its pharmacodynamic and pharmacokinetic characteristics. Therefore, by studying the differences in the main serum components, we can confirm the effectiveness of drugs and speculate about the treatment mechanism of these drugs. Based on the high-resolution mass spectrometric analysis, this experiment compared the differences in the serum principal components among the compound Sophora decoction, mixture of matrine, oxymatrine monomer, and indirubicone monomer and provided a modern scientific basis for elucidating the clinical efficacy of compound Sophora decoction.

\section{Materials and Methods}

2.1. Instruments. In this study, we used Shimadzu Prominence UPLC system LC-20 (Shimadzu, Japan), AB Sciex Triple QTOF 5600+ high-resolution mass spectrometer (AB Sciex, USA), BT25S 100,000th analytical balance (Saidoris, Switzerland), XW-80A vortex mixer (Jintan Yichen, China), KQ-500VD ultrasonic cleaner (Kunshan, China), TGL-16 desktop high-speed refrigerated centrifuge (Xiangyi, China), and UPT-II-10T ultrapure water machine (UPT, China).

2.2. Materials. Sophora flavescens Ait., Sanguisorba officinalis L., Bletilla striata Diels., Glycyrrhiza uralensis Fisch, and Indigo naturalis were authenticated by professor Keli Chen of the Hubei University of Traditional Chinese Medicine (Wuhan, China), and they complied with the provisions of the 2015 edition of the Chinese pharmacopeia. The reserved samples were stored in the herbarium of the Hubei Institute of Traditional Chinese Medicine (Wuhan, China); the voucher specimen numbers of Sophora flavescens Ait., Sanguisorba officinalis L., Bletilla striata Diels., Glycyrrhiza uralensis Fisch, and Indigo naturalis samples were 201809012, 201809004, 201809015, 201809020, and 201809009, respectively. The standard reference compounds, including indirubin (batch number: 110717200204), matrine (batch number: 110805-200508), and oxymatrine (batch number: 110780-201508), were purchased from China Food and Drug Identification Institute (Beijing, China); 5\% 2,4,6-trinitrobenzene sulfonic acid (TNBS, 3LBD6811V) was purchased from Sigma. Mesalazine granules (batch number: 170904) were purchased from the France Aifa Pharmaceutical group.

2.3. Experimental Animals and Treatment. A total of 28 Wistar rats (male, weight $190-210 \mathrm{~g}$ ) provided by the Beijing Weitong Lever Laboratory Animal Technology Co. (Beijing, China, License no. SCXK (Beijing) 2016-0006) were lodged under specific pathogen-free (SPF) conditions with free access to the autoclaved food and water in the experimental animal center of the Hubei Provincial Hospital of Traditional Chinese Medicine (HPHTCM, Wuhan, China). The animals were housed under the standard laboratory conditions at a temperature of $25 \pm 2^{\circ} \mathrm{C}$, the relative humidity of $50-55 \%$, and $12 \mathrm{~h}$ light/dark cycle and acclimatized to the environment for at least one week before animal experiments. The animal welfare and experimental procedures strictly complied with the guidelines of the Animal Research Institute Committee of HPHTCM (no. HBZYY-2018-043) and National Institutes of Health guidelines and regulations.

\subsection{Preparation of Compound Sophora Decoction (FFKST).} The compound Sophora decoction consists of Sophora flavescens Ait., Sanguisorba officinalis L., Bletilla striata Diels., Glycyrrhiza uralensis Fisch, and Indigo naturalis $(3: 3: 2: 2$ : 1). These herbs were soaked with water for $30 \mathrm{~min}$ and then boiled them twice for $1 \mathrm{~h}$ each time. Then, the supernatant was concentrated to $1.25 \mathrm{~g} / \mathrm{mL}$. Before using, it was diluted with saline to the dosage volume of $1 \mathrm{~mL}$.

2.5. Preparation of Matrine and Oxymatrine Intragastric Solution. According to the original prescription, a mixture of matrine and oxymatrine $(3: 1)$ was used to prepare the intragastric solution [8]. Before using, it was diluted with normal saline to the dosage volume of $1 \mathrm{~mL}$.

2.6. Grouping and Modeling of Animals. After 24 hours of fasting, the rats were anesthetized with $1 \%$ pentobarbital sodium (40 mg/kg), and then, 3 rats were randomly selected as the normal group. The remaining rats were induced to UC by administering with TNBS (5\% TNBS and 50\% ethanol solution were mixed in a proportion of $12: 5$, and the mixture $(4.25 \mathrm{ml} / \mathrm{kg})$ was injected into the rat colon through the anus with a rubber hose of $2 \mathrm{~mm}$ in diameter), and the normal group was injected into the colon with normal saline $(4.25 \mathrm{ml} / \mathrm{kg})$. Then, the rats were laid flat and provided with a free diet after they woke up naturally. When the rats developed loose stools, then the mucus and bloody stools 
appeared, accompanied by the loss of appetite, laziness, weight loss, arched back, and dull hair, indicating that the model was successfully induced $[9,10]$. The UC-induced rats were randomly divided into the model group $(\mathrm{M})$, positive control group (MS), compound Sophora decoction group (FFKST), matrine and oxymatrine group (KST), and indirubin group (DYH) with 5 rats in each group.

2.7. Drug Administration and Sampling. The positive control group (MS) was administered with $0.5 \mathrm{~g} / \mathrm{kg} / \mathrm{d}$ of mesalazine; the compound Sophora decoction group (FFKST) was administered with $5 \mathrm{~g} / \mathrm{kg} / \mathrm{d}$ of the crude drug; the matrine and oxymatrine group (KST) was administered with $10.83 \mathrm{mg} /$ $\mathrm{kg} / \mathrm{d}$ of matrine and $3.61 \mathrm{mg} / \mathrm{kg} / \mathrm{d}$ of oxymatrine solution [11] by gavage; the indirubin group (DYH) was administered with $14.44 \mathrm{mg} / \mathrm{kg} / \mathrm{d}$ of indirubin [12] solution by gavage. The blank group and model group (M) received the same amount of saline $(1 \mathrm{ml} / \mathrm{d})$. After seven days of continuous administration, $4 \mathrm{~mL}$ of blood was collected from the abdominal aorta of each rat from each group. The serum was separated and stored in the refrigerator at $-80^{\circ} \mathrm{C}$. After collecting the samples, all rats were euthanized by subjecting to the inhalation of excess $\mathrm{CO}_{2}$. Two rats from each group were randomly selected, and their colonic tissues were separated for the pathological examination after euthanizing.

2.8. Handling of Analytical Samples [13]. The serum samples were subjected to methanol precipitation. Precisely, $0.4 \mathrm{~mL}$ of drug-containing serum of the rats from each group was transferred to $1.2 \mathrm{~mL}$ of methanol (the volume ratio was 1 : 4), then scrolled for $1 \mathrm{~min}$, and centrifuged at $12000 \mathrm{r} / \mathrm{min}$ for $5 \mathrm{~min}$ at $4^{\circ} \mathrm{C}$. The supernatant was aspirated under dry nitrogen at $40^{\circ} \mathrm{C}$. Then, the residue was reconstituted with $200 \mu \mathrm{L}$ of methanol and sonicated in an ice bath for $10 \mathrm{~min}$. Finally, it was centrifuged at $12000 \mathrm{r} / \mathrm{min}$ for $10 \mathrm{~min}$, and the supernatant was used as the test product.

2.9. Chromatographic Conditions. Agilent Eclipse XDB C18 chromatographic column $(100 \mathrm{~mm} \times 2.1 \mathrm{~mm}, 3.5 \mathrm{~m})$ was used for separation. The mobile phase was water $(\mathrm{A})$ and methanol (B) $(0.1 \%$ formic acid) gradient elution, $0-3 \mathrm{~min}$ (B: $20 \%-40 \%$ ), 3-10 $\min$ (B: 40\%-54\%), 10-25 $\min$ (B: $54 \%$ ), 25-35 $\mathrm{min}$ (B: $54 \%-70 \%), \quad 35-45 \mathrm{~min}$ (B: $70 \%-80 \%$ ), 45-50 min (B: 80\%), 50-60 min (B: 80\%-100\%), 70-72 min (B: $100 \%-20 \%$ ), and $72-77 \mathrm{~min}$ (B: $20 \%$ ); flow rate, $300 \mu \mathrm{L} /$ min; column temperature, $40^{\circ} \mathrm{C}$; and injection volume, $10 \mu \mathrm{L}$. One QC sample was injected into HPLC for instrument calibration among every three test samples, and a total number of 5 QC points were set.

2.10. Mass Spectrometry Conditions. ESI source was selected, and scanning was performed in the positive and negative ion modes. The scanning range was $70-1500 \mathrm{~m} / z$; ion-source gas 1 (GS1): 55 psi; ion-source gas 2 (GS2): 60 psi; CUR: 30 psi; ion-source temperature (TEM): $550^{\circ} \mathrm{C}$; ion-source voltage (ISVF): $5500 \mathrm{~V} /-4500 \mathrm{~V}$; decluster voltage (DP): $100 \mathrm{~V}$; collision energy (CE): $35 \mathrm{~V} /-35 \mathrm{~V}$; collision energy gain (CES): $15 \mathrm{~V} /-15 \mathrm{~V}$; and data acquisition mode: IDA.

2.11. Analytical Procedure $[14,15]$. The high-resolution data were comparatively analyzed. In MarkView 1.3, each set of data was imported, multiple sets of principal component analysis were performed, and the molecular weights and retention times of the substances with large differences $(P<0.01$ or $P<0.05)$ were derived based on the cluster analysis information. The above information was imported into PeakView 2.0, the molecular compositions of the differential substances were determined by the first-level mass spectrometry, and the molecular structural formula of the substance was determined by the second-level mass spectrometry combined with ChemSpider (https://www. chemspider.com). During qualitative analysis, the top 8000 compounds $(P<0.01$ or $P<0.05)$ were analyzed individually, and the isotope peaks were selected from the firstlevel mass spectrum, which was related to the second-level mass spectrum in the order of molecular weight error $(<5 \mathrm{ppm})$, and the molecular formulas of these components were imported into ChemSpider and input in the software in the mol2 format to perform docking.

\section{Results}

3.1. Histopathological Observation of Colon. As shown in Figure 1, the colonic structure of the normal group was clear, and the mucosal epithelium was intact and continuous. Contrarily, the colonic structure was destroyed in the model group (M) with multiple mucosal erosion, punctate necrosis, and hemorrhage. The mucosa in the positive control group (MS) was intact like the mucosa of a newborn. The colons of the compound Sophora decoction group (FFKST) and the indirubin group (DYH) were similar to that of the positive control group, while the colons of the matrine and oxymatrine group (KST) were comparable to that of the model group $(M)$.

\subsection{Analysis of Differences in Serum Principal Components of} Each Group. After all, the above course was processed (including all the samples were processed, they were analyzed in the positive and negative ion modes firstly, and then, the high-resolution data were comparatively analyzed by the software). The processing results obtained by MarkView are shown in Figures 2 and 3. Generally, the absorption, distribution, and metabolism of drugs could inevitably cause changes in the main components of serum. Therefore, the differences in the main components of serum from each group were studied to understand the effects of drugs on endogenous substances. MarkView, special software for metabolomic studies, includes an independent PCA plug-in, which can be used to compare the differences of the main components in two or more groups simultaneously. As shown in figure, the clustering effect of the scatter distribution of different groups in the negative ion mode is obvious. The compound Sophora decoction group (FFKST) is spatially close to the positive control group (MS). The 


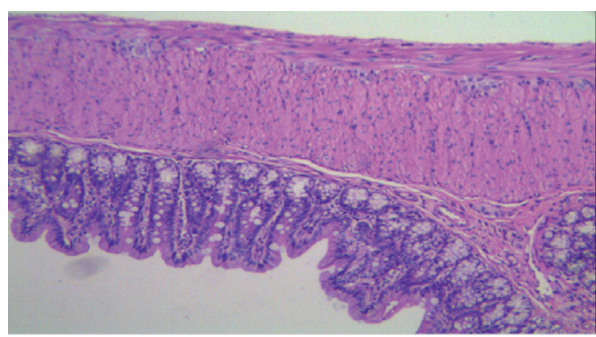

(a)

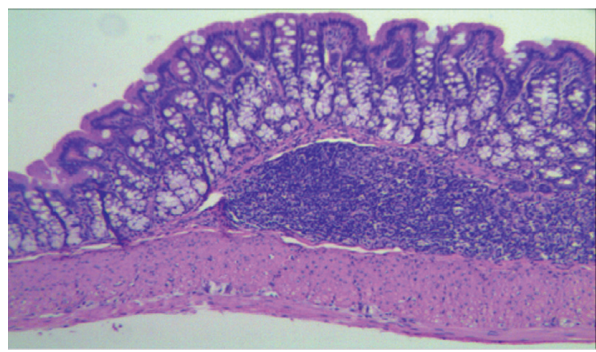

(c)

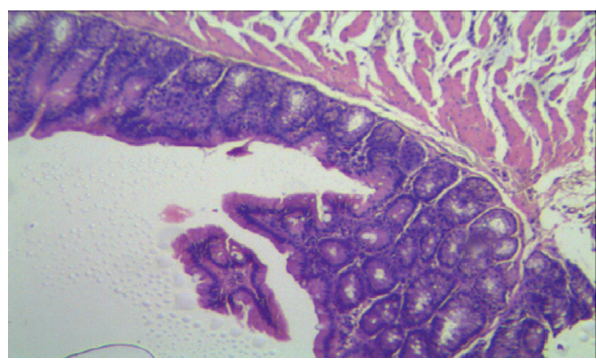

(e)

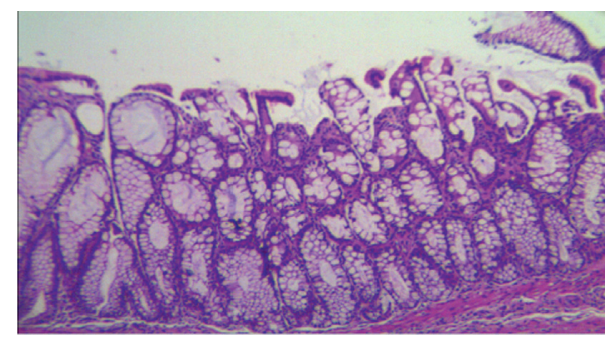

(b)

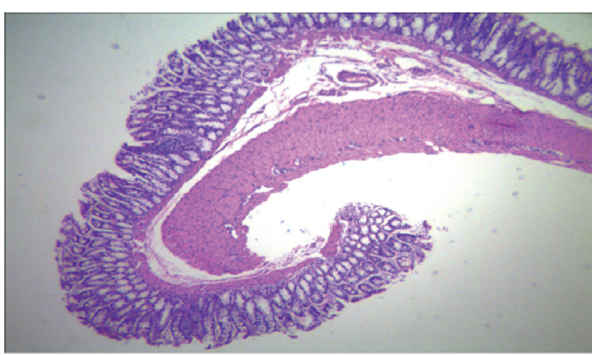

(d)

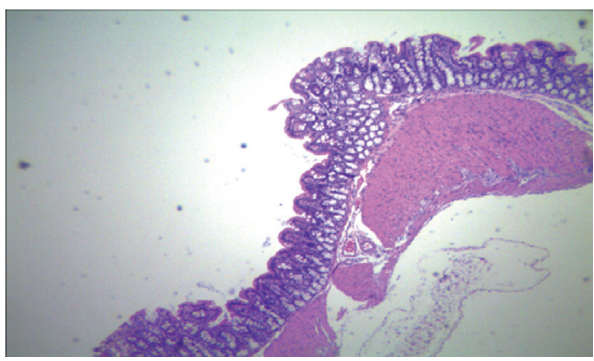

(f)

FIgURE 1: Pathological changes of colonic mucosa (HE, ×40): (a) normal group; (b) M group; (c) MS group; (d) FFKST group; (e) KST group; (f) DYH group.

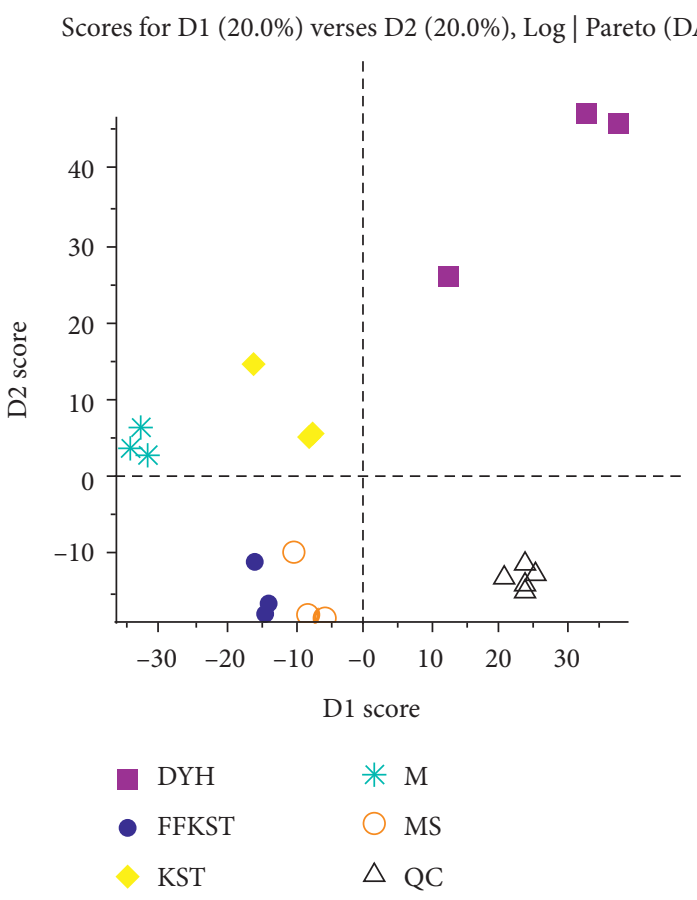

FIGURE 2: Cluster analysis result obtained by MarkView in negative ion mode (results were output by the software MarkView 1.3).

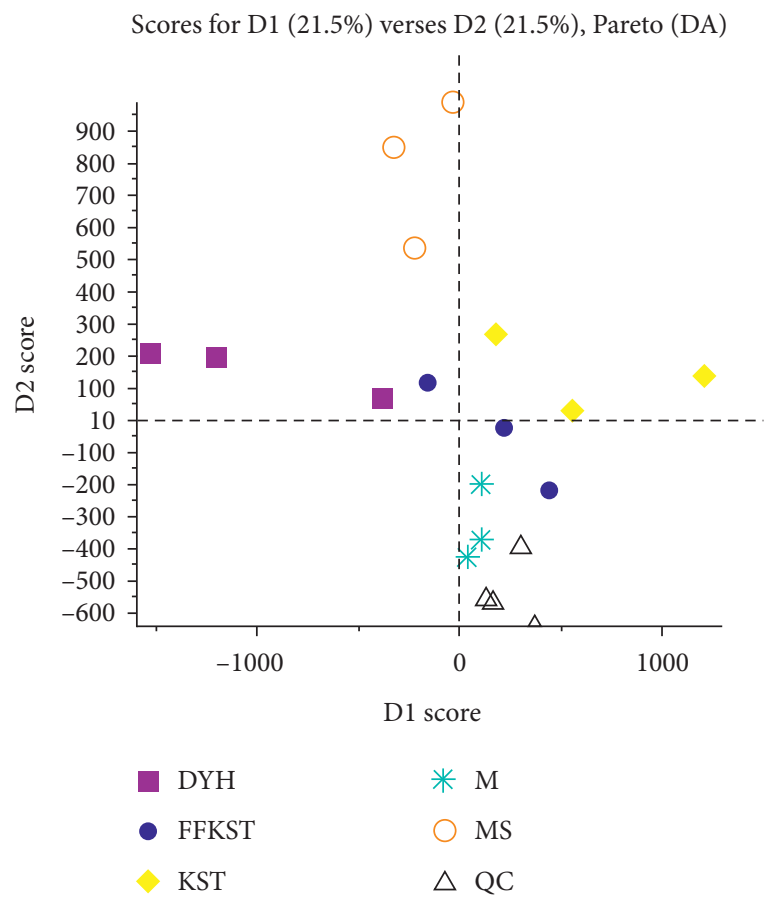

FIgURE 3: Cluster analysis result obtained by MarkView in positive ion mode (results were output by the software MarkView 1.3). 


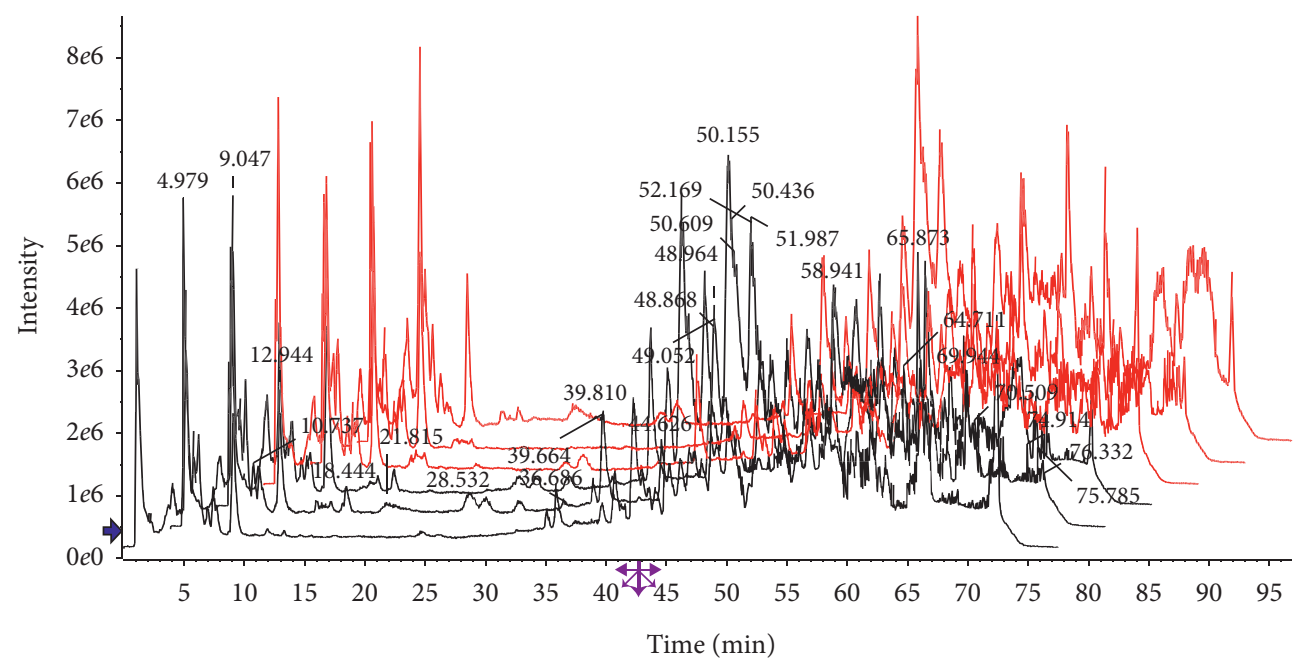

- TIC from Data201901042.wiff (sample 1) - FFKST3

- TIC from Data201901043.wiff (sample 1) - FFKST14

- TIC from Data201901044.wiff (sample 1) - FFKST28

- TIC from Data2019010414.wiff (sample 1) - M19

- TIC from Data2019010415.wiff (sample 1) - M4

○ TIC from Data2019010416.wiff (sample 1) - M47

FIgURE 4: Superposition diagram of the total ion flow of FFKST and $M$ in the negative ion mode.

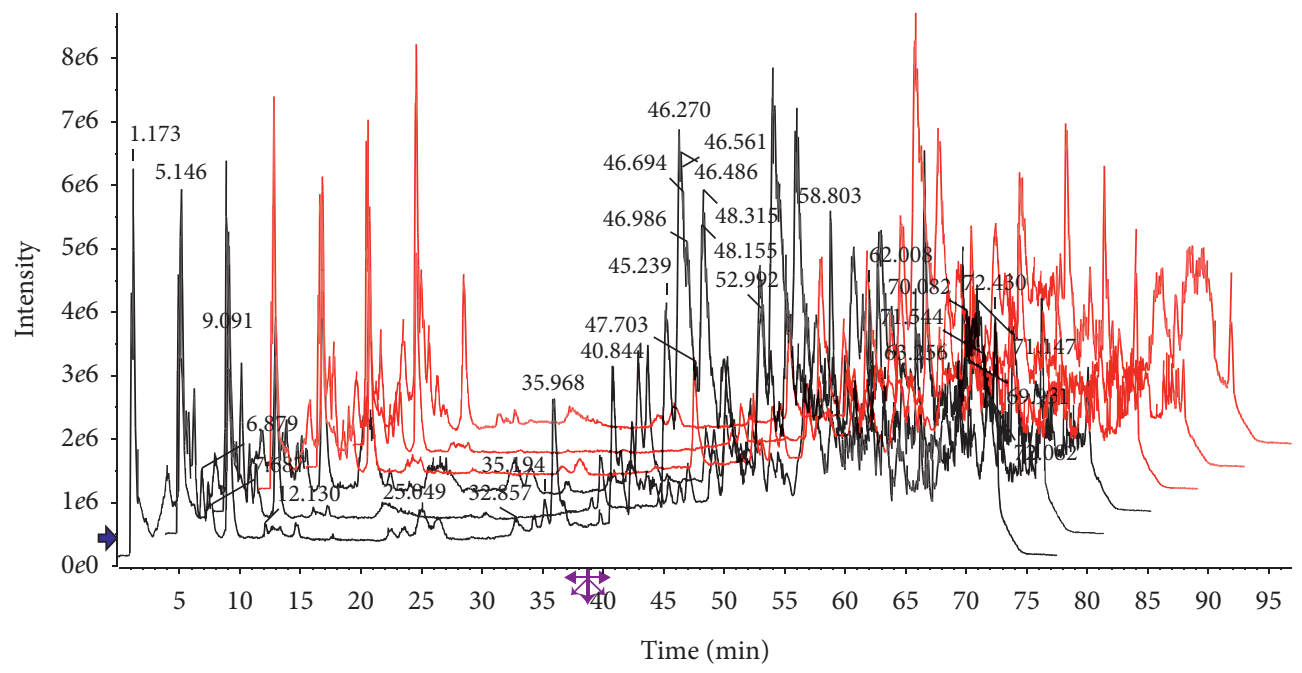

- TIC from Data2019010410.wiff (sample 1) - KST48

- TIC from Data2019010411.wiff (sample 1) - KST24

- TIC from Data2019010412.wiff (sample 1) - KST13

- TIC from Data2019010414.wiff (sample 1) - M19

- TIC from Data2019010415.wiff (sample 1) - M4

- TIC from Data2019010416.wiff (sample 1) - M47

FIGURE 5: Superposition diagram of total ion flow of KST and M in the negative ion mode.

matrine and oxymatrine group (KST) is spatially close to the model group (M), but the two of which could still be distinguished. The indirubin group (DYH) is located alone in another space. The results showed that, after the administration of compound Sophora decoction, the main components of serum in the model animals were close to those 
TABLE 1: Details of the differential compounds between FFKST and M in the negative ion mode.

\begin{tabular}{|c|c|c|c|c|c|c|}
\hline $\begin{array}{l}\text { Rt } \\
(\mathrm{min})\end{array}$ & Compounds & Formula & $\underset{\text { experimental }}{[\mathrm{M}-\mathrm{H}]}$ & $\underset{\text { calculated }}{[\mathrm{M}-\mathrm{H}]}$ & $\begin{array}{l}\text { Error } \\
(\mathrm{ppm})\end{array}$ & MS/MS \\
\hline 2.71 & Unknown & $\mathrm{C}_{9} \mathrm{H}_{10} \mathrm{O}_{3}$ & 165.0559 & 165.0557 & 1.10 & 121,96 \\
\hline 3.23 & 4-Methoxybenzenesulfonic acid & $\mathrm{C}_{7} \mathrm{H}_{8} \mathrm{O}_{4} \mathrm{~S}$ & 187.0072 & 187.0071 & 0.80 & 107,79 \\
\hline 4.87 & 2-(4-Hydroxybutyl)succinic acid & $\mathrm{C}_{8} \mathrm{H}_{14} \mathrm{O}_{5}$ & 189.0770 & 189.0768 & 0.80 & $145,129,127,99$ \\
\hline 10.65 & $N$-Boc-4-piperidineethanol & $\mathrm{C}_{12} \mathrm{H}_{23} \mathrm{NO}_{3}$ & 228.1607 & 228.1605 & 0.80 & 211,167 \\
\hline 3.42 & 4-Methylcatechol-O,O-diacetic acid & $\mathrm{C}_{11} \mathrm{H}_{12} \mathrm{O}_{6}$ & 239.0559 & 239.0561 & -0.90 & $179,177,149$ \\
\hline 50.09 & Palmitelaidic acid & $\mathrm{C}_{16} \mathrm{H}_{30} \mathrm{O}_{2}$ & 253.2169 & 253.2173 & -1.60 & 235 \\
\hline 2.01 & Piscidic acid & $\mathrm{C}_{11} \mathrm{H}_{12} \mathrm{O}_{7}$ & 255.0505 & 255.051 & -2.10 & $175,165,149,135,93$ \\
\hline 4.20 & Benzyl D-glucopyranuronate & $\mathrm{C}_{13} \mathrm{H}_{16} \mathrm{O}_{7}$ & 283.0820 & 283.0823 & -1.20 & 107,87 \\
\hline 18.17 & [6]-Gingerol & $\mathrm{C}_{17} \mathrm{H}_{26} \mathrm{O}_{4}$ & 293.1753 & 293.1758 & -1.80 & $\begin{array}{c}236,221,205,192 \\
177\end{array}$ \\
\hline 42.04 & Ricinoleic acid & $\mathrm{C}_{18} \mathrm{H}_{34} \mathrm{O}_{3}$ & 297.2427 & 297.2435 & -2.80 & 279,183 \\
\hline 36.27 & Hexanedioic acid, dihexyl ester & $\mathrm{C}_{18} \mathrm{H}_{34} \mathrm{O}_{4}$ & 313.2375 & 313.2384 & -3.00 & $\begin{array}{c}295,277,213,201 \\
171,000\end{array}$ \\
\hline 50.09 & Unknown & $\mathrm{C}_{15} \mathrm{H}_{26} \mathrm{~N}_{6} \mathrm{O}_{2}$ & 321.2040 & 321.2044 & -1.40 & 253,235 \\
\hline 59.35 & Unknown & $\mathrm{C}_{18} \mathrm{H}_{36} \mathrm{~N}_{6}$ & 335.2926 & 335.2929 & -0.80 & 130,97 \\
\hline 56.21 & Unknown & $\mathrm{C}_{15} \mathrm{H}_{33} \mathrm{~N}_{3} \mathrm{O}_{7}$ & 366.2240 & 366.2246 & -1.60 & 281 \\
\hline 27.85 & $\begin{array}{c}\text { 12-[2-(3-Carboxypropoxy)phenoxy] } \\
\text { dodecanoic acid }\end{array}$ & $\mathrm{C}_{22} \mathrm{H}_{34} \mathrm{O}_{6}$ & 393.2287 & 393.2296 & -2.30 & 257,231 \\
\hline 7.62 & Unknown & $\mathrm{C}_{17} \mathrm{H}_{17} \mathrm{~N}_{8} \mathrm{O}_{6} \mathrm{P}$ & 459.0916 & 459.0936 & -4.30 & $283,255,240$ \\
\hline 23.78 & Unknown & $\mathrm{C}_{25} \mathrm{H}_{32} \mathrm{O}_{8}$ & 459.2023 & 459.2024 & -0.30 & $323,254,186$ \\
\hline 43.02 & Unknown & $\mathrm{C}_{16} \mathrm{H}_{33} \mathrm{~N}_{8} \mathrm{O}_{6} \mathrm{P}$ & 463.2189 & 463.2188 & 0.20 & $378,163,78$ \\
\hline 29.71 & Glycocholic acid & $\mathrm{C}_{26} \mathrm{H}_{43} \mathrm{NO}_{6}$ & 464.3017 & 464.3018 & -0.10 & 402,74 \\
\hline 23.57 & Unknown & $\mathrm{C}_{22} \mathrm{H}_{38} \mathrm{~N}_{2} \mathrm{O}_{9}$ & 473.2503 & 473.2505 & -0.30 & 405,361 \\
\hline 40.70 & Unknown & $\mathrm{C}_{21} \mathrm{H}_{39} \mathrm{~N}_{3} \mathrm{O}_{9}$ & 476.2610 & 476.2614 & -0.70 & 391,251 \\
\hline 58.36 & $\begin{array}{c}3,6,9,12,15 \text {-Pentaoxaheptacos-1-yl- } \\
\text { hydrogen sulfate }\end{array}$ & $\mathrm{C}_{22} \mathrm{H}_{46} \mathrm{O}_{9} \mathrm{~S}$ & 485.2792 & 485.279 & 0.50 & $469,421,280$ \\
\hline 24.86 & Unknown & $\mathrm{C}_{23} \mathrm{H}_{44} \mathrm{NO}_{6} \mathrm{PS}$ & 492.2560 & 492.2554 & 1.20 & 407 \\
\hline 6.61 & Unknown & $\mathrm{C}_{19} \mathrm{H}_{10} \mathrm{~N}_{10} \mathrm{O}_{8}$ & 505.0612 & 505.061 & 0.30 & $329,314,298$ \\
\hline 61.95 & Unknown & $\mathrm{C}_{31} \mathrm{H}_{37} \mathrm{~N}_{3} \mathrm{O}_{4}$ & 514.2716 & 514.2711 & 0.90 & $485,470,116$ \\
\hline 40.71 & Unknown & $\mathrm{C}_{21} \mathrm{H}_{31} \mathrm{~N}_{13} \mathrm{O}_{5}$ & 544.2482 & 544.2498 & -3.00 & $476,391,129$ \\
\hline
\end{tabular}

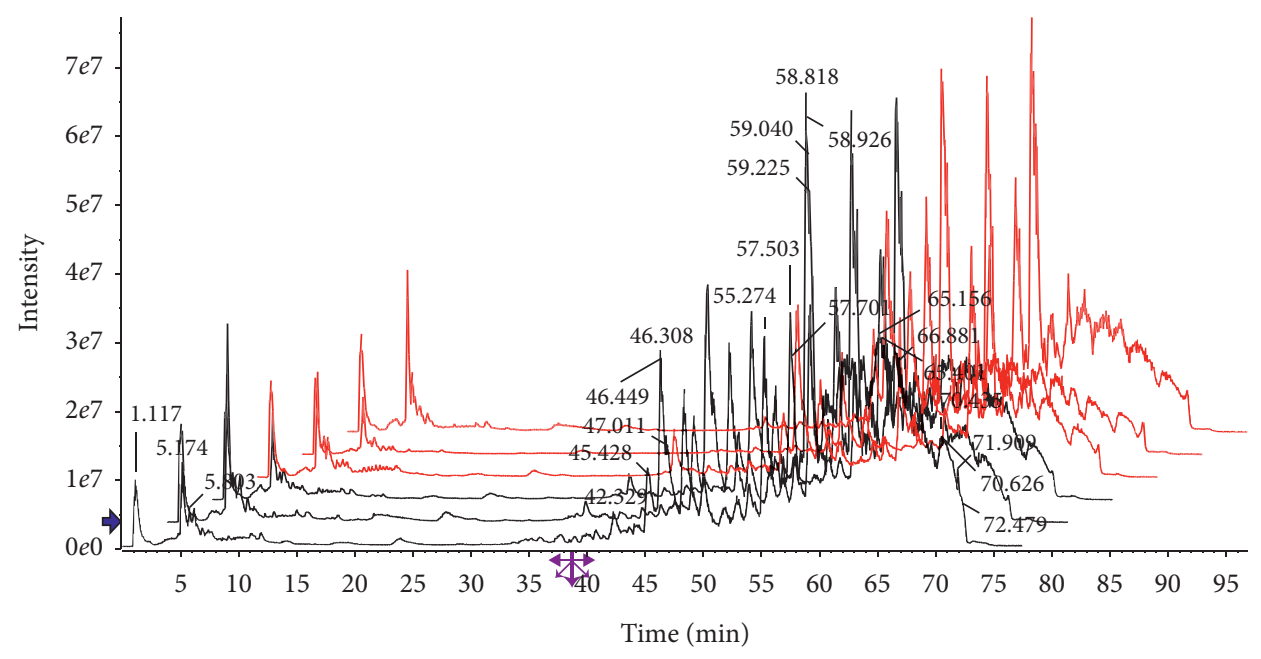

- TIC from Data201901022.wiff (sample 1) - FFKST3

- TIC from Data201901026.wiff (sample 1) - FFKST14

- TIC from Data201901027.wiff (sample 1) - FFKST28

- TIC from Data2019010217.wiff (sample 1) - M19

- TIC from Data2019010218.wiff (sample 1) - M4

- TIC from Data2019010219.wiff (sample 1) - M47

FIgURE 6: Superposition diagram of total ion flow of FFKST and M in the positive ion mode. 
TABLE 2: Details of differential compounds between KST and $M$ in the negative ion mode.

\begin{tabular}{|c|c|c|c|c|c|c|}
\hline $\begin{array}{l}\text { Rt } \\
(\mathrm{min})\end{array}$ & Compounds & Formula & $\underset{\text { experimental }}{[\mathrm{M}-\mathrm{H}]}$ & $\begin{array}{l}{[\mathrm{M}-\mathrm{H}]} \\
\text { calculated }\end{array}$ & $\begin{array}{l}\text { Error } \\
(\mathrm{ppm})\end{array}$ & MS/MS \\
\hline 7.68 & Butalbital & $\mathrm{C}_{11} \mathrm{H}_{16} \mathrm{~N}_{2} \mathrm{O}_{3}$ & 223.109 & 223.1088 & 0.8 & 180,153 \\
\hline 31.13 & $\begin{array}{c}\text { 6-(3-Hydroxypentan-3-yl)-2,2,6-trimethyloxane-3-carboxylic } \\
\text { acid }\end{array}$ & $\mathrm{C}_{14} \mathrm{H}_{26} \mathrm{O}_{4}$ & 257.1759 & 257.1758 & 0.3 & 239,195 \\
\hline 57.88 & Stearic acid & $\mathrm{C}_{18} \mathrm{H}_{36} \mathrm{O}_{2}$ & 283.2641 & 283.2643 & -0.50 & 265 \\
\hline 7.68 & $3^{\prime}$-Azido-2 ${ }^{\prime}, 3^{\prime}$-dideoxyguanosine & $\mathrm{C}_{10} \mathrm{H}_{12} \mathrm{~N}_{8} \mathrm{O}_{3}$ & 291.0962 & 291.096 & 0.8 & $\begin{array}{l}291,223 \\
180\end{array}$ \\
\hline 18.24 & [6]-Gingerol & $\mathrm{C}_{17} \mathrm{H}_{26} \mathrm{O}_{4}$ & 293.1762 & 293.1758 & 1.30 & $\begin{array}{l}236,221 \\
205,192 \\
177\end{array}$ \\
\hline 39.1 & Unknown & $\mathrm{C}_{14} \mathrm{H}_{24} \mathrm{~N}_{6} \mathrm{O}_{2}$ & 307.1892 & 307.1888 & 1.3 & $\begin{array}{l}259,239 \\
102\end{array}$ \\
\hline 7.66 & 2-(\{5-Chloro-2-[(2-fluorobenzyl)oxy]benzyl $\}$ amino $)$ ethanol & $\mathrm{C}_{16} \mathrm{H}_{17} \mathrm{NO}_{2} \mathrm{FCl}$ & 308.0863 & 308.0859 & 1.3 & 223,180 \\
\hline 51.95 & Unknown & $\mathrm{C}_{14} \mathrm{H}_{26} \mathrm{~N}_{6} \mathrm{O}_{2}$ & 309.2045 & 309.2044 & 0.2 & 241,104 \\
\hline 44.5 & Octadecanedioic acid & $\mathrm{C}_{18} \mathrm{H}_{34} \mathrm{O}_{4}$ & 313.2385 & 313.2384 & 0.2 & 295,251 \\
\hline 47.53 & 2-Dodecylbenzenesulfonic acid & $\mathrm{C}_{18} \mathrm{H}_{30} \mathrm{O}_{3} \mathrm{~S}$ & 325.1843 & 325.1843 & 0 & 293,183 \\
\hline 27.82 & 12-[2-(3-Carboxypropoxy)phenoxy]dodecanoic acid & $\mathrm{C}_{22} \mathrm{H}_{34} \mathrm{O}_{6}$ & 393.2282 & 393.2283 & -0.2 & 257,231 \\
\hline 37.12 & Glycodeoxycholic acid & $\mathrm{C}_{26} \mathrm{H}_{43} \mathrm{NO}_{5}$ & 448.3062 & 448.3068 & -1.4 & 404,74 \\
\hline 29.67 & Glycocholic acid & $\mathrm{C}_{26} \mathrm{H}_{43} \mathrm{NO}_{6}$ & 464.3013 & 464.3018 & -1 & 402,74 \\
\hline 4.19 & Unknown & $\mathrm{C}_{19} \mathrm{H}_{34} \mathrm{~N}_{6} \mathrm{O}_{10}$ & 505.2261 & 505.2264 & 3.00 & $\begin{array}{l}306,241 \\
207\end{array}$ \\
\hline 60.82 & $\begin{array}{c}\text { 2-Deoxy-4-O-[(2E,4R,6E,8E,10S,11R,12S)-11-hydroxy- } \\
\text { 2,4,6,8,10,12-hexamethyl-5-oxo-2,6,8-docosatrienoyl]-D- } \\
\text { erythro-pentonic acid }\end{array}$ & $\mathrm{C}_{33} \mathrm{H}_{56} \mathrm{O}_{8}$ & 579.3901 & 579.3902 & -0.2 & $\begin{array}{c}403,175 \\
113\end{array}$ \\
\hline 46.14 & Unknown & $\mathrm{C}_{26} \mathrm{H}_{48} \mathrm{~N}_{7} \mathrm{O}_{9} \mathrm{P}$ & 632.3177 & 632.3178 & -0.2 & $\begin{array}{l}504,279 \\
112 \\
536,426\end{array}$ \\
\hline 61.95 & Galactosylceramide & $\mathrm{C}_{40} \mathrm{H}_{77} \mathrm{NO}_{8}$ & 698.558 & 698.5576 & 0.5 & $\begin{array}{c}290,179 \\
89\end{array}$ \\
\hline 45.06 & Unknown & $\mathrm{C}_{28} \mathrm{H}_{59} \mathrm{~N}_{3} \mathrm{O}_{13} \mathrm{P}_{4}$ & 768.2929 & 768.2926 & 0.4 & $\begin{array}{c}248,180 \\
112\end{array}$ \\
\hline
\end{tabular}

TABLE 3: Details of differential compounds between FFKST and $M$ in the positive ion mode.

\begin{tabular}{|c|c|c|c|c|c|c|}
\hline $\begin{array}{l}\text { Rt } \\
(\min )\end{array}$ & Compounds & Formula & $\underset{\text { experimental }}{[\mathrm{M}+\mathrm{H}]}$ & $\underset{\text { calculated }}{[\mathrm{M}+\mathrm{H}]}$ & $\begin{array}{l}\text { Error } \\
(\mathrm{ppm})\end{array}$ & MS/MS \\
\hline 60.41 & Docosanamid & $\mathrm{C}_{22} \mathrm{H}_{45} \mathrm{NO}$ & 340.3565 & 340.3574 & -2.6 & $\begin{array}{c}102,88 \\
287,243,215,\end{array}$ \\
\hline 62.02 & (20R)-Cholesta-3,5-dien & $\mathrm{C}_{27} \mathrm{H}_{44}$ & 369.3501 & 369.3516 & -4 & $\begin{array}{l}175,161,147 \\
109,95\end{array}$ \\
\hline 37.99 & Val-his-ala-gly & $\mathrm{C}_{16} \mathrm{H}_{26} \mathrm{~N}_{6} \mathrm{O}_{5}$ & 383.2033 & 383.2037 & -1.2 & $327,281,267$ \\
\hline 41.45 & $\begin{array}{l}\text { Propyl-6-O-(2-acetamido-2-deoxy- } \beta \text {-D-glucopyranosyl)- } \\
\text { 2-amino- } 2 \text {-deoxy- } \alpha \text {-D-glucopyranoside }\end{array}$ & $\mathrm{C}_{17} \mathrm{H}_{32} \mathrm{~N}_{2} \mathrm{O}_{10}$ & 425.2118 & 425.213 & -2.8 & 355,281 \\
\hline 60.21 & $\begin{array}{c}\text { 4-[(2-Cyanoethyl)amino]-3-hydroxy-2,2-dimethyl-4- } \\
\text { oxobutyl-palmitate }\end{array}$ & $\mathrm{C}_{25} \mathrm{H}_{46} \mathrm{~N}_{2} \mathrm{O}_{4}$ & 439.3536 & 439.353 & 1.3 & 407 \\
\hline 60.73 & $\begin{array}{l}\text { 3-(1-Hydroxy-2,4-dimethylhexyl)-2-isopropoxy-5,6- } \\
\text { dimethoxy-4-pyridinyl diisopropylcarbamate }\end{array}$ & $\mathrm{C}_{25} \mathrm{H}_{44} \mathrm{~N}_{2} \mathrm{O}_{6}$ & 469.3272 & 469.3272 & 0 & 329,189 \\
\hline 62.21 & $\begin{array}{c}N \text {-[(1S,2S,8S,8aS)-7-\{(2S)-1-[Bis(2-methoxyethyl)amino]- } \\
\text { 1-oxo-2-propanyl }\} \text {-8-hydroxy-1,4a-dimethyldecahydro- } \\
\text { 2-naphthalenyl]-3,3-dimethylbutanamide }\end{array}$ & $\mathrm{C}_{27} \mathrm{H}_{50} \mathrm{~N}_{2} \mathrm{O}_{5}$ & 483.3782 & 483.3792 & -2.2 & 453 \\
\hline 62.39 & $\begin{array}{c}N-[(9 Z)-9-\text { Octadecen-1-yl]-2-oxohexadecanamide } \\
\text { (2R,4S,5S,7S)-5-Amino- } N \text {-butyl-7- }\{4-[4-\end{array}$ & $\mathrm{C}_{34} \mathrm{H}_{65} \mathrm{NO}_{2}$ & 520.5056 & 520.5088 & -6.2 & $282,264,252$ \\
\hline 61.88 & $\begin{array}{c}\text { (dimethylamino)butoxy]-3-(3-methoxypropoxy)benzyl\}- } \\
\text { 4-hydroxy-2,8-dimethylnonanamide }\end{array}$ & $\mathrm{C}_{32} \mathrm{H}_{59} \mathrm{~N}_{3} \mathrm{O}_{5}$ & 566.4512 & 566.4527 & -2.7 & $226,184,104$ \\
\hline 60.86 & $\begin{array}{l}\text { L-Valyl-L-isoleucylglycyl-L-alanyl- } N \text {-[(2S)-6-amino-1- } \\
\text { oxo-2-hexanyl]-L-lysinamide }\end{array}$ & $\mathrm{C}_{28} \mathrm{H}_{54} \mathrm{~N}_{8} \mathrm{O}_{6}$ & 599.4247 & 599.4239 & 1.3 & 300 \\
\hline 63.68 & $\begin{array}{l}\text { L-Lysyl-L-seryl-L-leucyl-N5-(diaminomethylene)-L- } \\
\text { ornithyl-L-seryl-L-phenylalanyl-L-lysine }\end{array}$ & $\mathrm{C}_{39} \mathrm{H}_{68} \mathrm{~N}_{12} \mathrm{O}_{10}$ & 865.5265 & 865.5254 & -1.2 & $\begin{array}{c}780,721,597 \\
575,145.00\end{array}$ \\
\hline
\end{tabular}




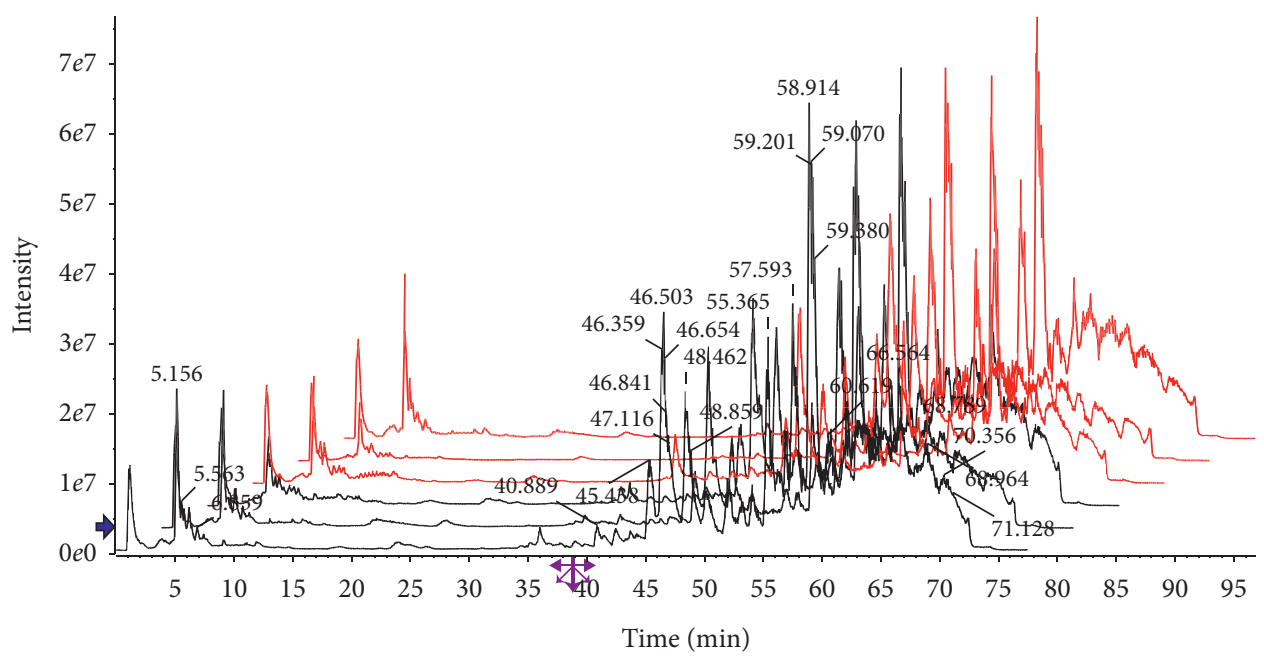

- TIC from Data2019010213.wiff (sample 1) - KST48

- TIC from Data2019010214.wiff (sample 1) - KST24

- TIC from Data2019010215.wiff (sample 1) - KST13

- TIC from Data2019010217.wiff (sample 1) - M19

- TIC from Data2019010218.wiff (sample 1) - M4

- TIC from Data2019010219.wiff (sample 1) - M47

Figure 7: Superposition diagram of total ion flow of KST and $\mathrm{M}$ in the positive ion mode.

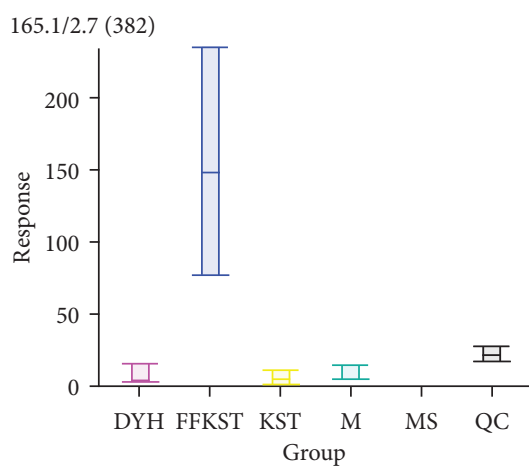

(a)

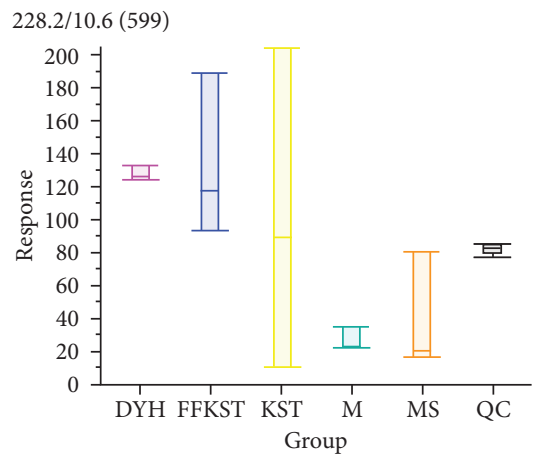

(d)

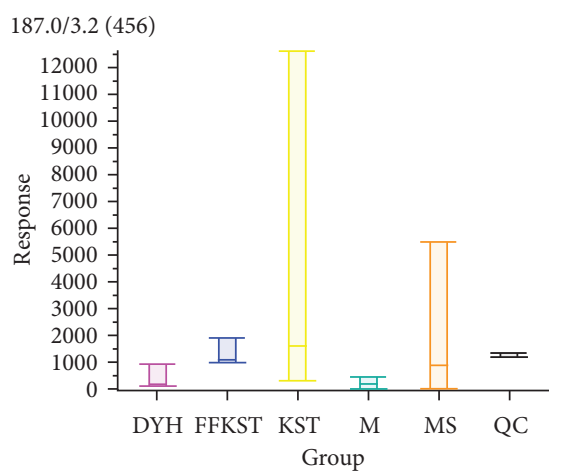

(b)

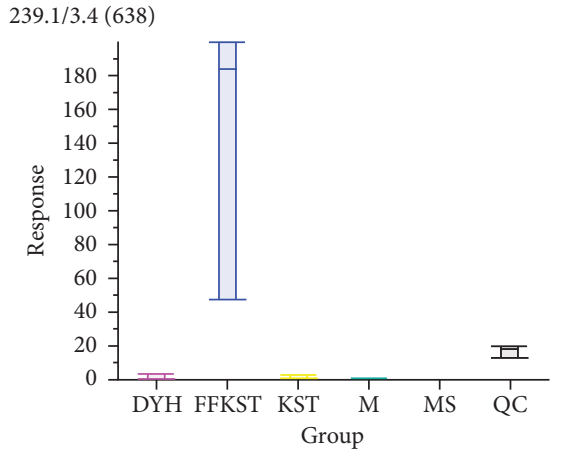

(e)

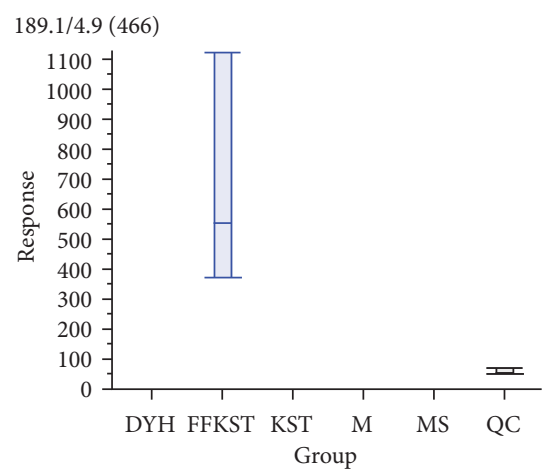

(c)

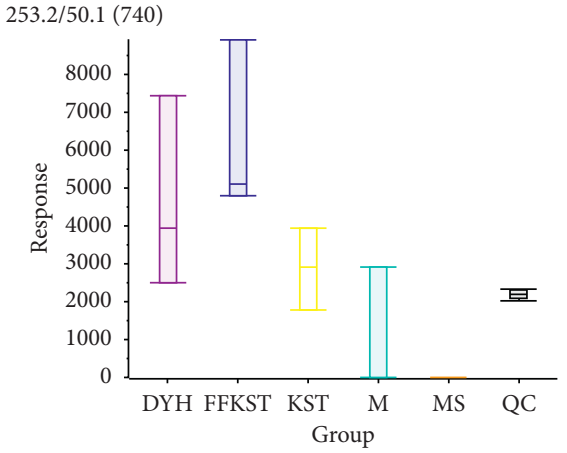

(f) 


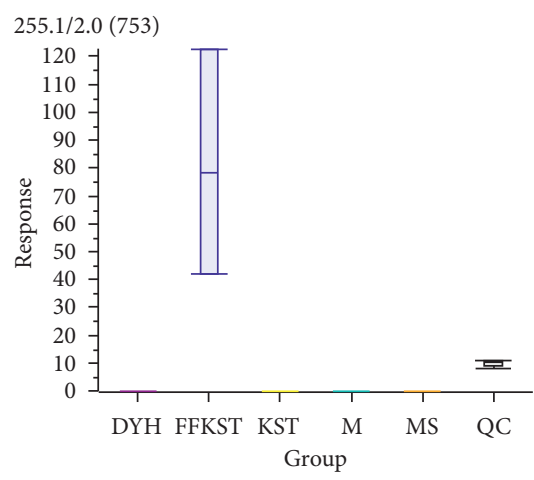

(g)

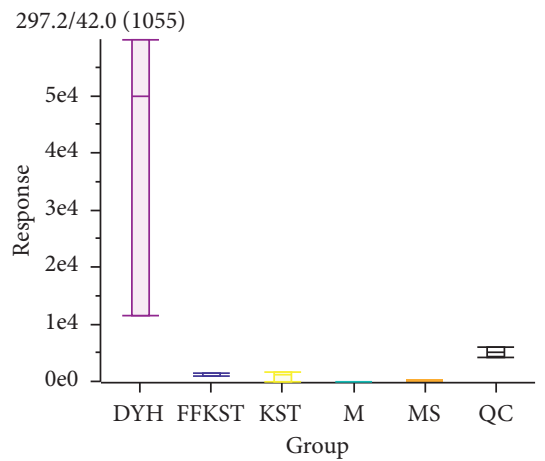

(j)

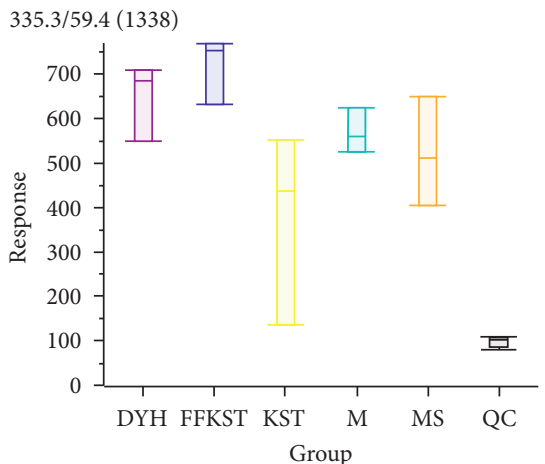

(m)

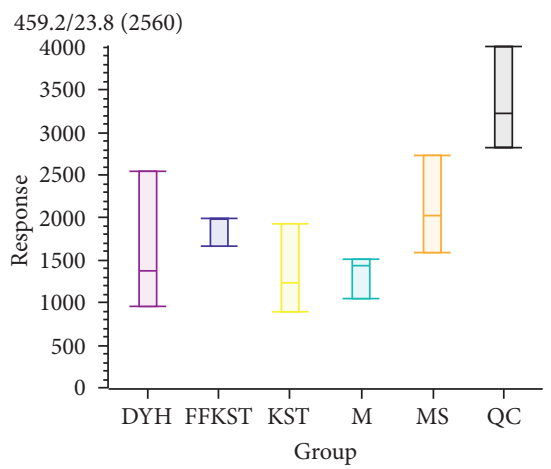

(p)

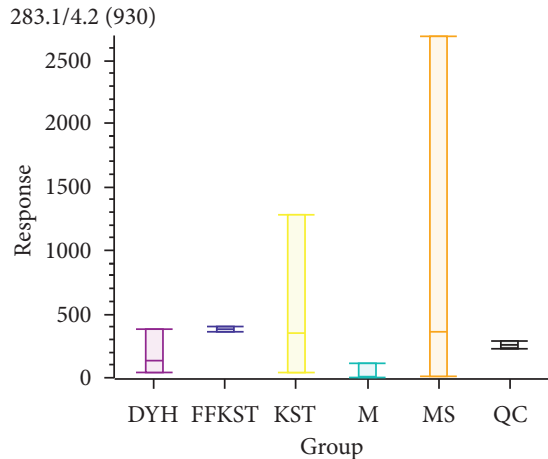

(h)

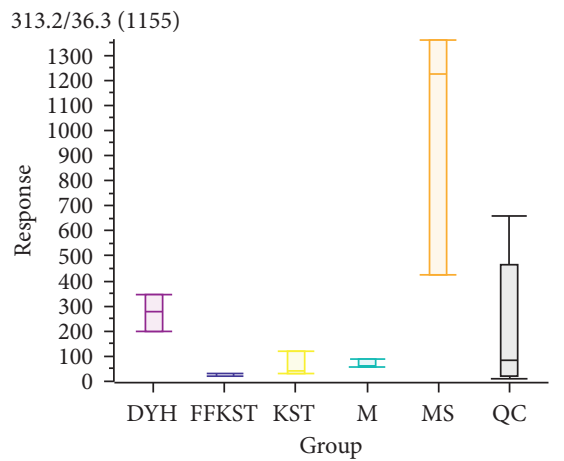

(k)

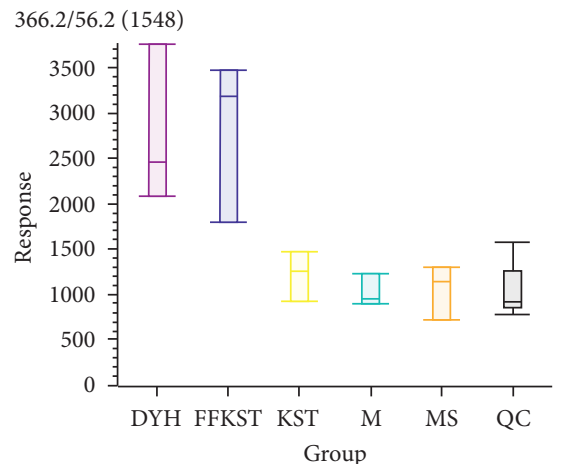

(n)

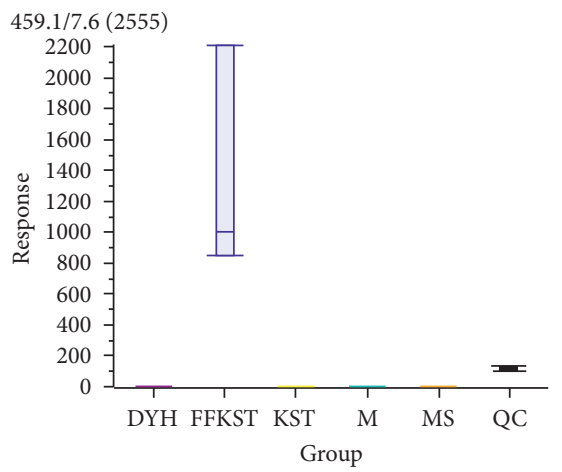

(q)

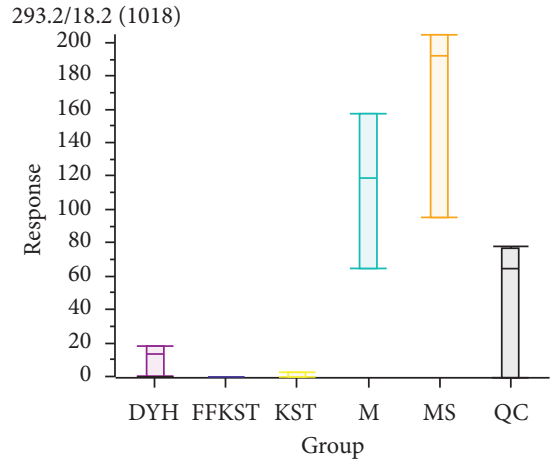

(i)

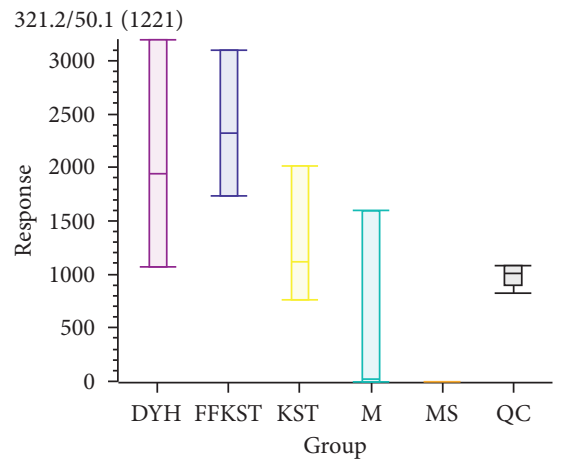

(1)

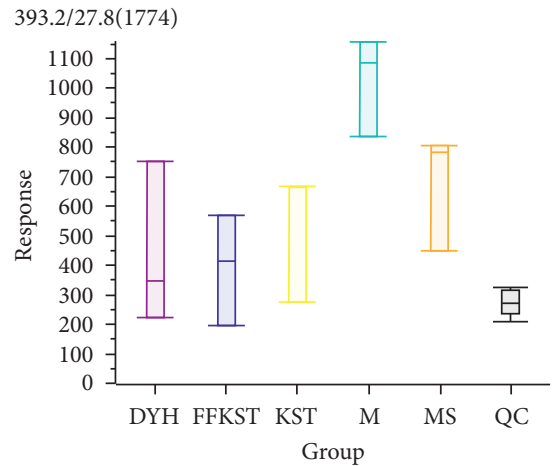

(o)

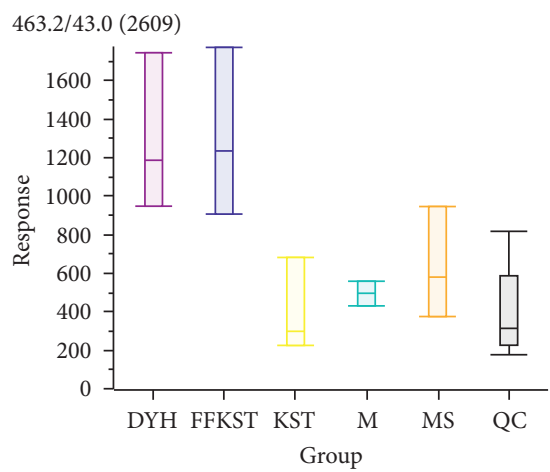

(r)

Figure 8: Continued. 


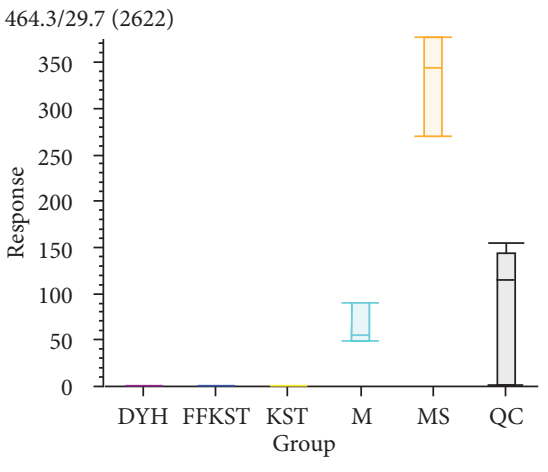

(s)

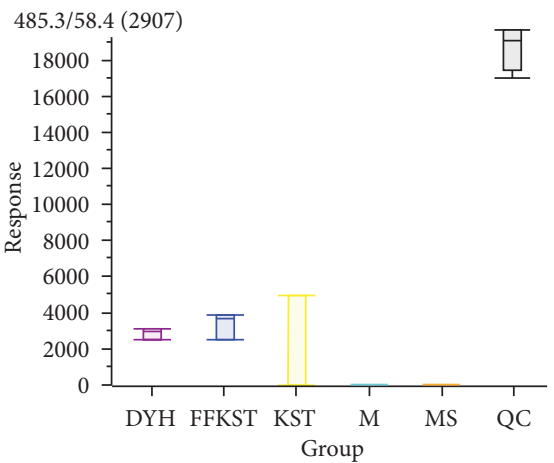

(v)

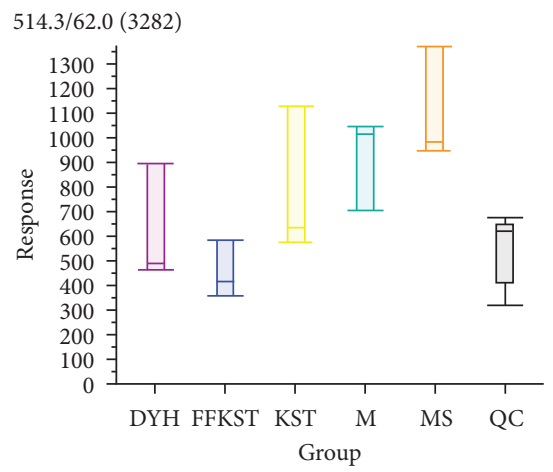

(y)

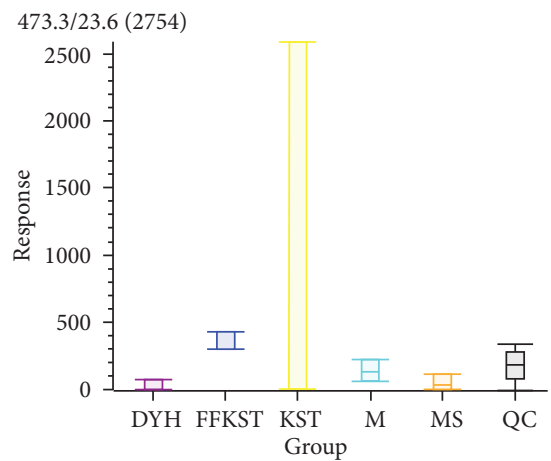

$(\mathrm{t})$

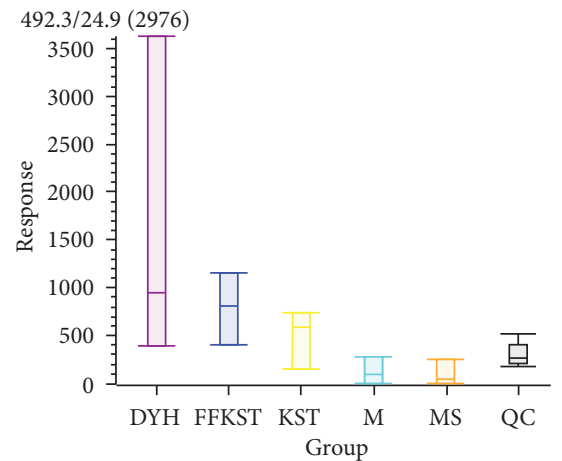

(w)

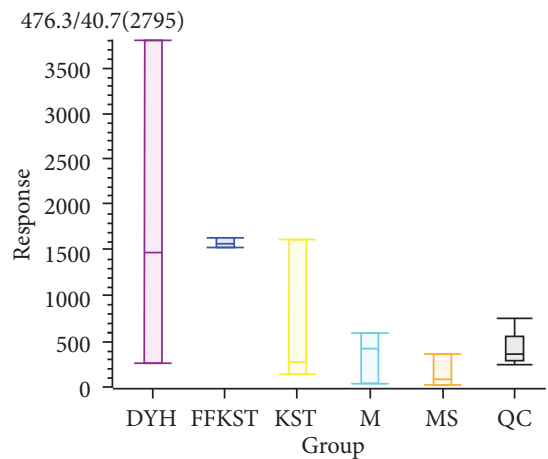

(u)

$$
\text { Group }
$$

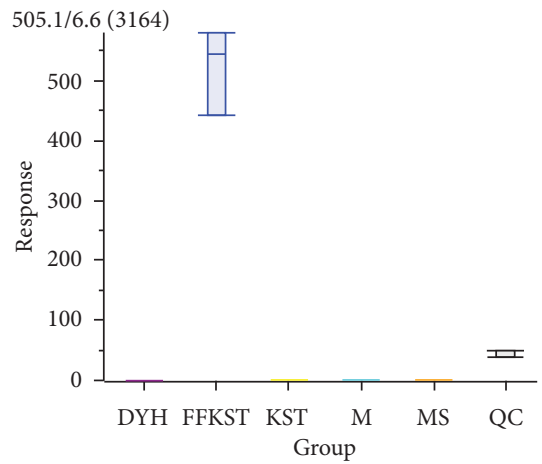

$(\mathrm{x})$

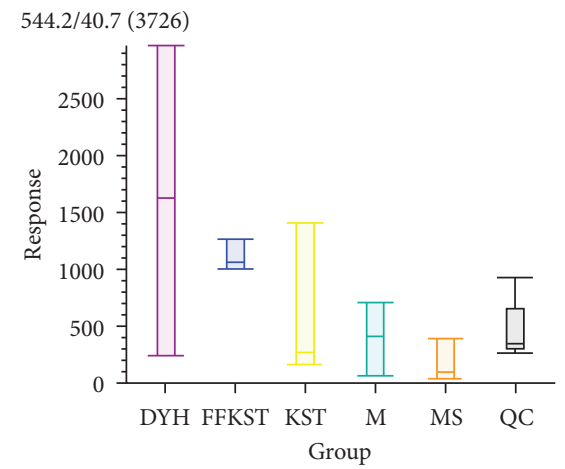

$(\mathrm{z})$

Figure 8: The response signals of 26 differential compounds in FFKST compared to $\mathrm{M}$ in each group $(P<0.01)$ in the negative ion mode (results were output by the software MarkView 1.3).

after the positive intervention. Compound Sophora decoction played a more consistent role in regulating the serum principal components than the monomer groups. A compound dose of matrine and oxymatrine monomer mixtures did not inflict major changes in the serum principal components of UC-induced rats, and the effects of those mixtures on the serum principal components were almost the same as the model group in terms of category and content without drug intervention. Certainly, differences were also observed between the KST and M groups, so the two mixtures could be distinguished in two dimensions to a very small degree. However, after the indirubin monomers produced a large difference in the serum principal components, the indirubin monomers, separated from the compound, regulated the serum principal components differently than the compound, causing a larger difference, and higher separation was achieved in the clustering results. All of the above was highly consistent with the pathological manifestations of colonic mucosa. In the positive ion mode, different groups failed to distribute the scattered points sufficiently, and the clustering effect was not significant, indicating that the substances, enriched in each group, overlapped in the positive ion mode. In the positive and negative modes, the five samples in the quality control group 


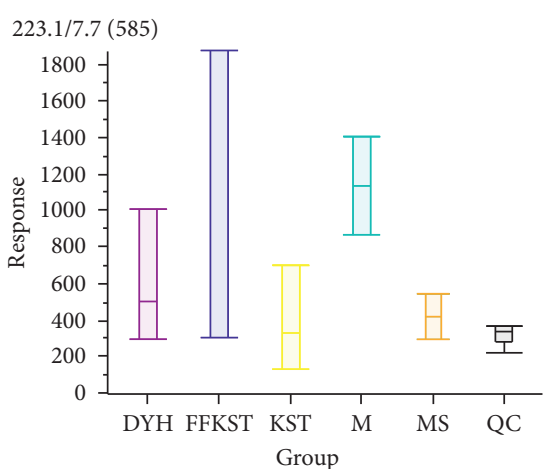

(a)

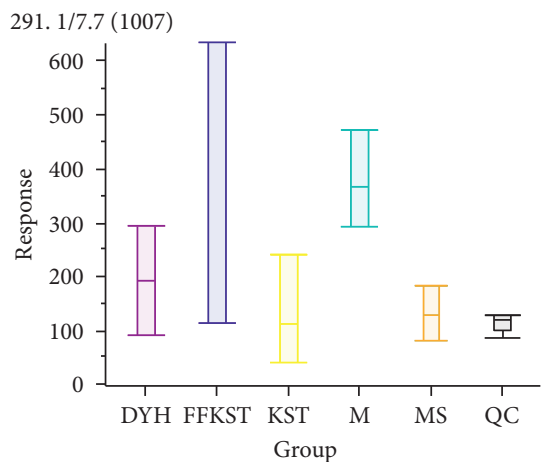

(d)

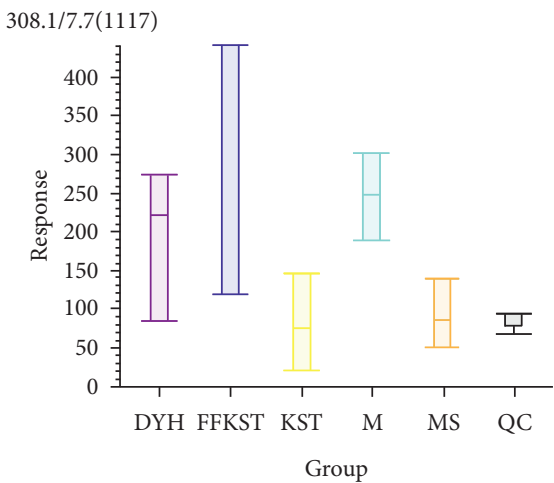

(g)

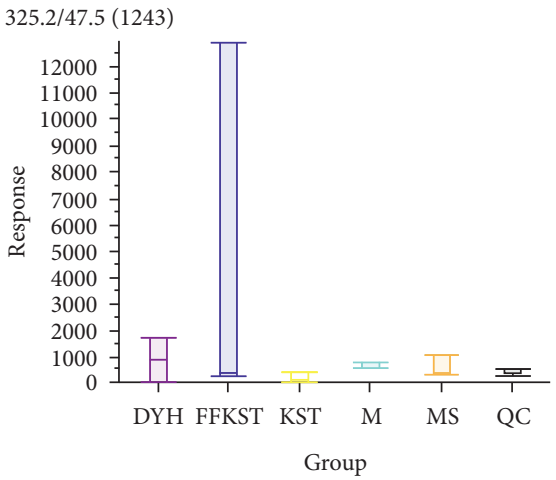

(j)

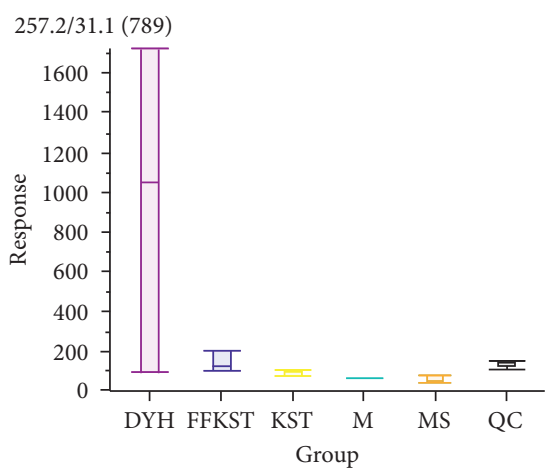

(b)

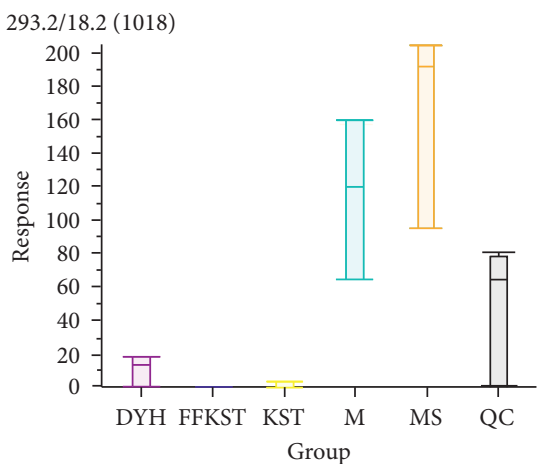

(e)

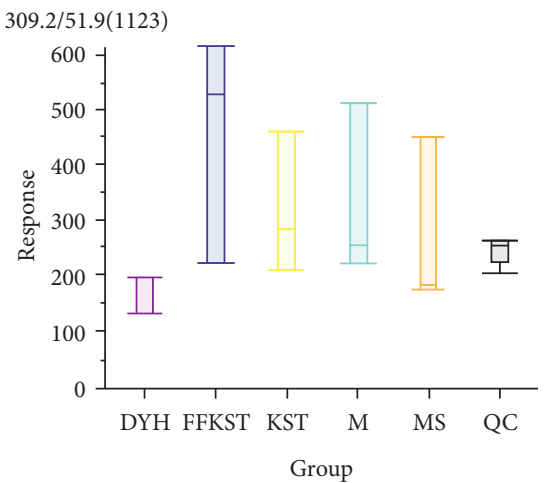

(h)

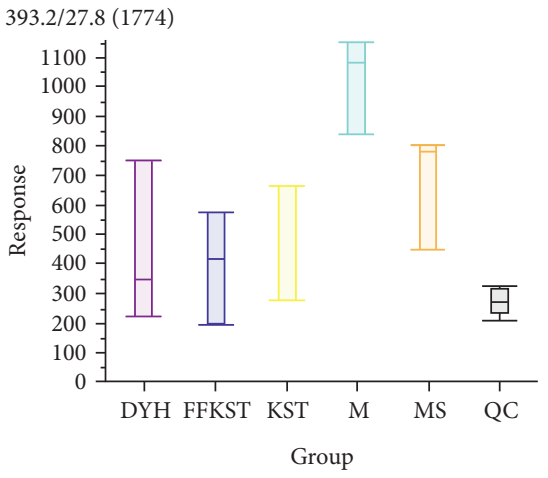

(k)

FIgURE 9: Continued.

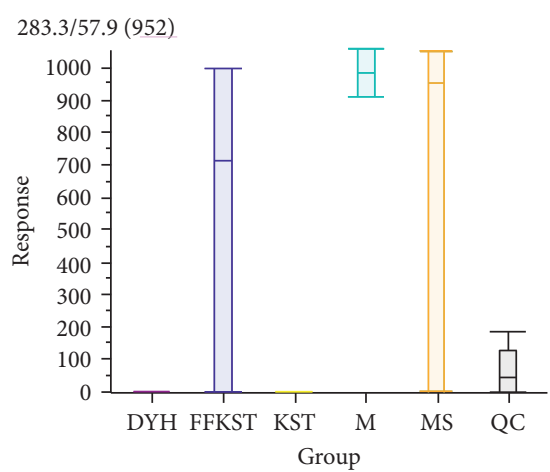

(c)

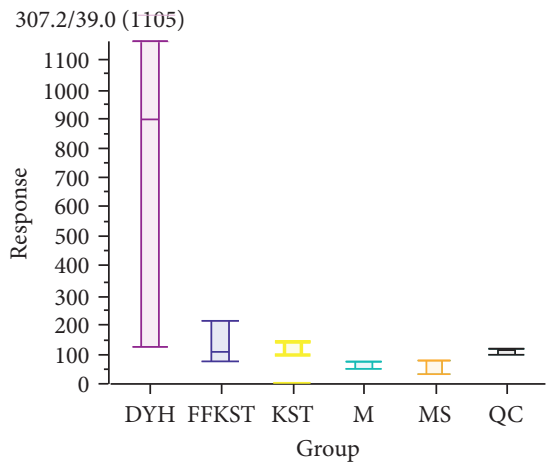

(f)

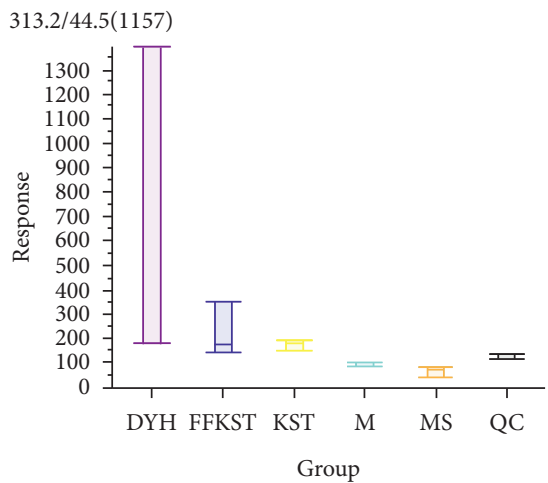

(i)

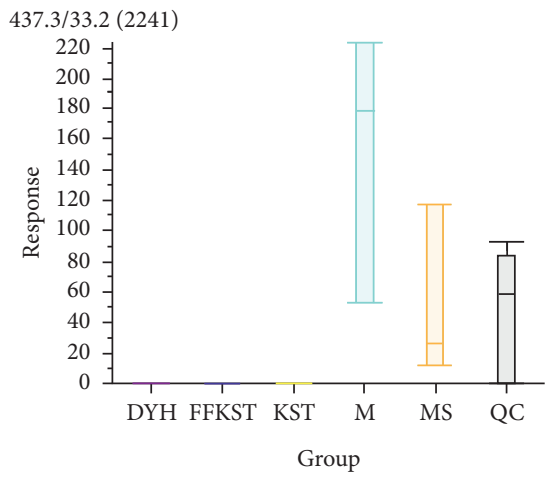

(l) 


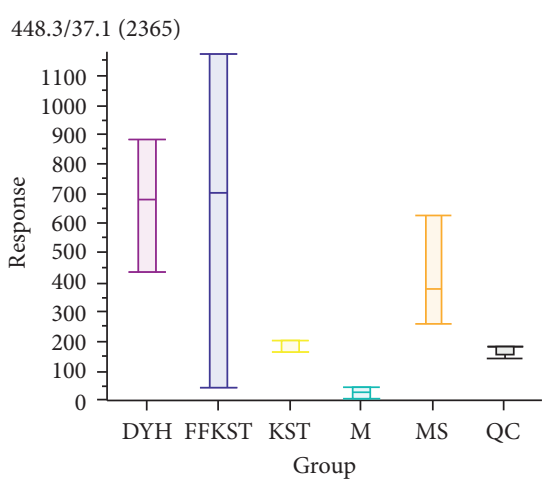

(m)

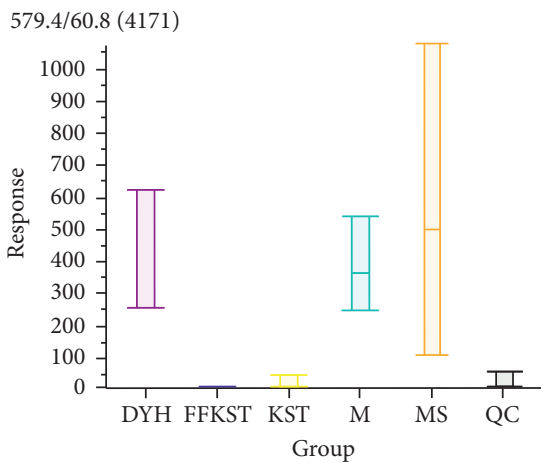

(p)

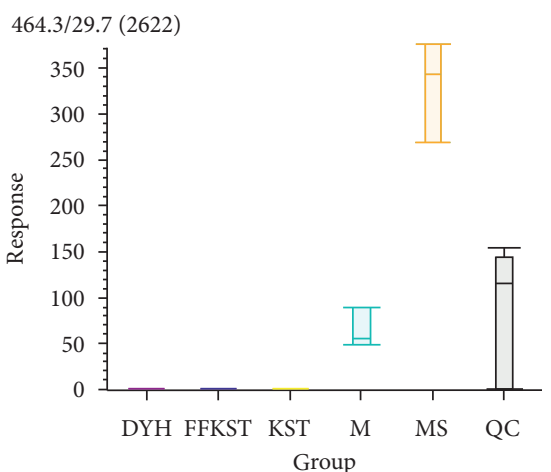

(n)

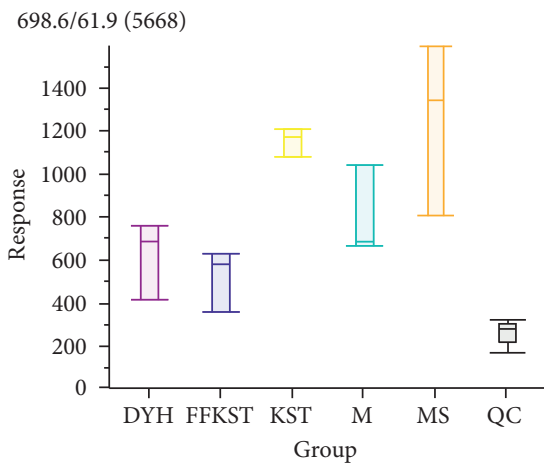

(q)

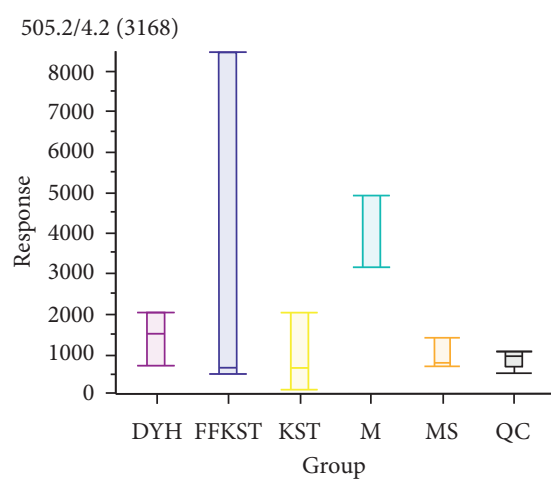

(o)

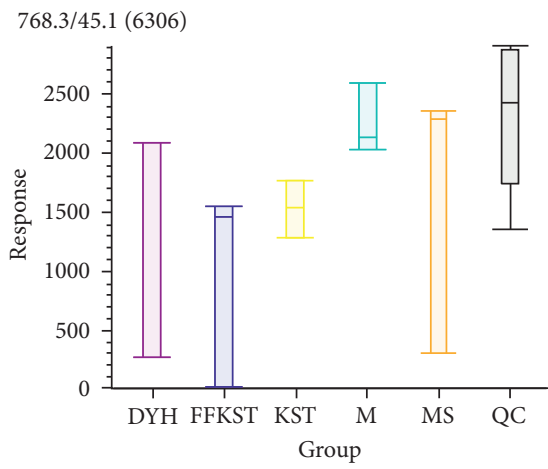

$(\mathrm{r})$

FIgURE 9: The response signals of 18 differential compounds in KST compared to $\mathrm{M}$ in each group $(P<0.01)$ in the negative ion mode (results were output by the software MarkView 1.3).

TABLE 4: Details of differential compounds between KST and M in the positive ion mode.

\begin{tabular}{|c|c|c|c|c|c|c|}
\hline $\begin{array}{l}\text { Rt } \\
(\min )\end{array}$ & Compounds & Formula & $\underset{\text { experimental }}{[\mathrm{M}+\mathrm{H}]}$ & $\underset{\text { calculated }}{[\mathrm{M}+\mathrm{H}]}$ & $\begin{array}{l}\text { Error } \\
(\mathrm{ppm})\end{array}$ & MS/MS \\
\hline 3.94 & $\begin{array}{l}\text { 3-(4-Hydroxypiperidin-1-yl-methyl)benzimidic acid ethyl } \\
\text { ester }\end{array}$ & $\mathrm{C}_{15} \mathrm{H}_{22} \mathrm{~N}_{2} \mathrm{O}_{2}$ & 263.1756 & 263.1754 & 0.7 & $245,162,96$ \\
\hline 23.97 & $\begin{array}{c}\text { 7-(2-Hydroxy-3-isopropoxypropyl)-3-methyl-8-[(2E)-2-(1- } \\
\text { phenylethylidene)hydrazino]-3,7-dihydro- } 1 \mathrm{H} \text {-purine-2,6- } \\
\text { dione }\end{array}$ & $\mathrm{C}_{20} \mathrm{H}_{26} \mathrm{~N}_{6} \mathrm{O}_{4}$ & 415.2096 & 415.2088 & 2.3 & $135,119,91$ \\
\hline 9.1 & Tyr-ser-asp-ile & $\mathrm{C}_{22} \mathrm{H}_{32} \mathrm{~N}_{4} \mathrm{O}_{9}$ & 497.2262 & 497.2242 & 4 & $\begin{array}{c}427,357,337 \\
271\end{array}$ \\
\hline 5.22 & Unknown & $\mathrm{C}_{48} \mathrm{H}_{82} \mathrm{~N}_{12} \mathrm{O}_{21}$ & 582.2928 & 582.2932 & -0.6 & $\begin{array}{l}961,582,217 \\
85\end{array}$ \\
\hline 5.12 & $\begin{array}{l}\text { 1-Oleoyl-2-(4Z,7Z,10Z,13Z,16Z,19Z-docosahexaenoyl)-sn- } \\
\text { glycerol }\end{array}$ & $\mathrm{C}_{43} \mathrm{H}_{70} \mathrm{O}_{5}$ & 667.5266 & 667.5296 & -4.6 & 383,341 \\
\hline 63.68 & $\begin{array}{l}\text { L-Lysyl-L-seryl-L-leucyl-N5-(diaminomethylene)-L- } \\
\text { ornithyl-L-seryl-L-phenylalanyl-L-lysine }\end{array}$ & $\mathrm{C}_{39} \mathrm{H}_{68} \mathrm{~N}_{12} \mathrm{O}_{10}$ & 865.5265 & 865.5254 & -1.2 & $\begin{array}{r}780,721,597 \\
575,145.00\end{array}$ \\
\hline 69.89 & Unknown & $\mathrm{C}_{44} \mathrm{H}_{78} \mathrm{~N}_{18} \mathrm{O}_{19}$ & 1163.576 & 1163.576 & -0.2 & 1074 \\
\hline
\end{tabular}

(QC) were closely placed, and the clustering effect was obvious, indicating that the results were stable and reproducible.

3.3. Identification of Differential Substances [16, 17]. According to the analytical procedure, the top 8000 compounds with the most significant differences were found in the compound Sophora decoction group (FFKST) and the matrine and oxymatrine group (KST) through pairwise comparisons to the model group (M). The superposition maps of total ion flow between compound Sophora decoction group (FFKST) and the model group (M) and matrine and oxymatrine group (KST) and the model group $(\mathrm{M})$, are shown in Figures 4-7, which reflect a visual difference between the target group and the model group. The identification results are shown in Tables $1-4$. In the negative ion mode, 26 differential compounds were identified in 


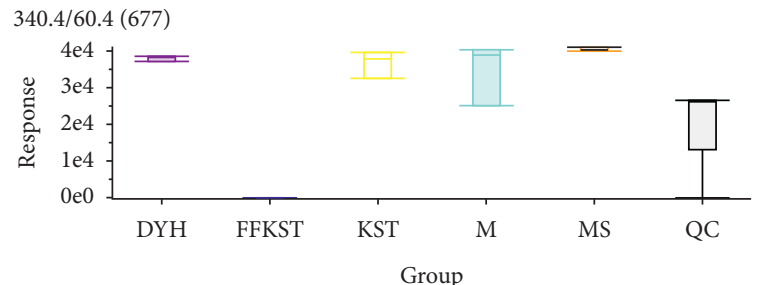

(a)

$383.4 / 37.9(1047)$

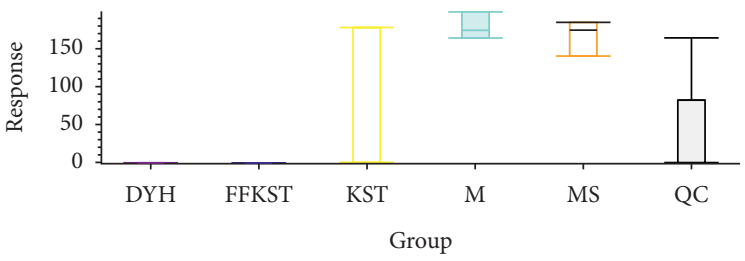

(c)

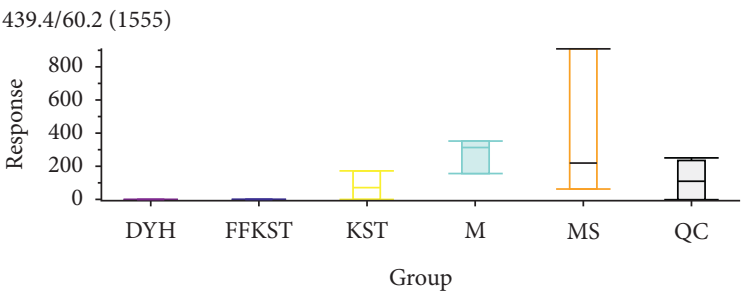

(e)

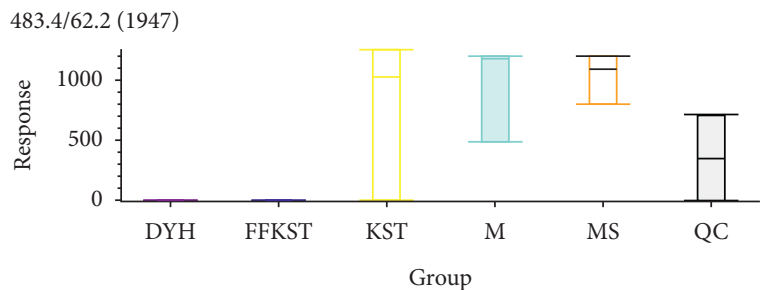

(g)

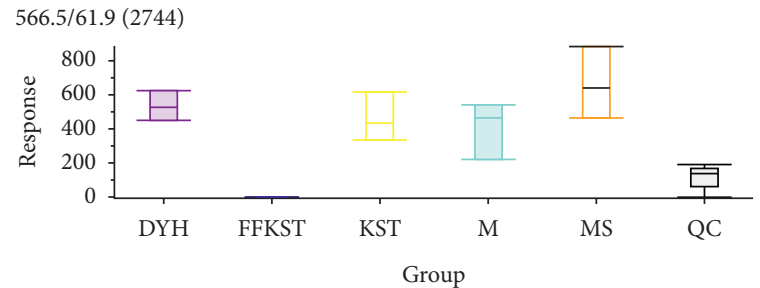

(i)

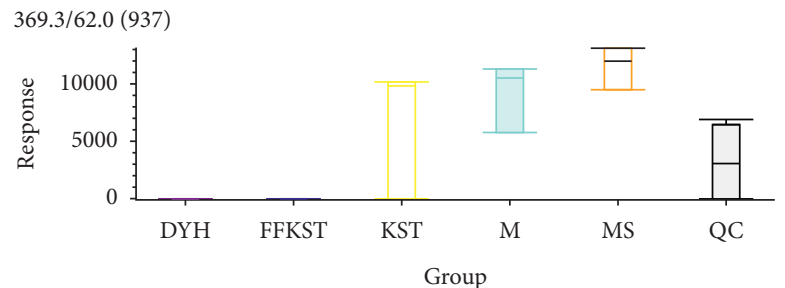

(b)

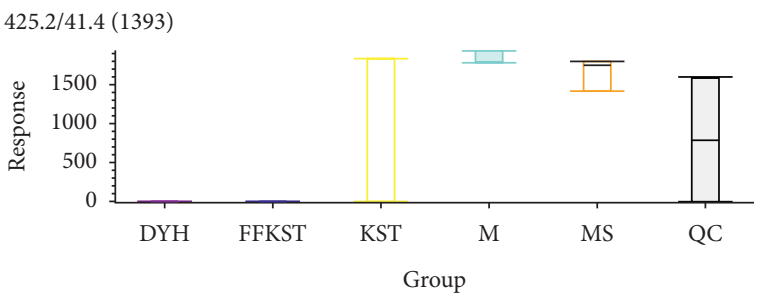

(d)

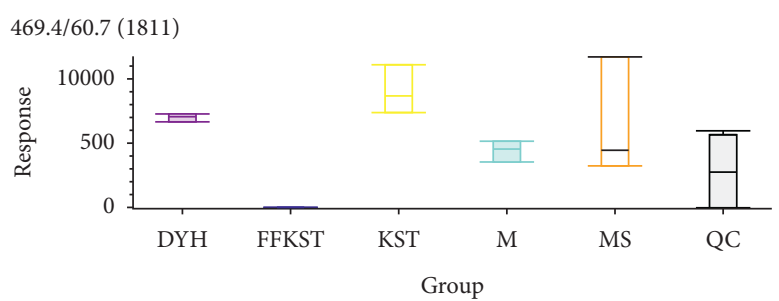

(f)

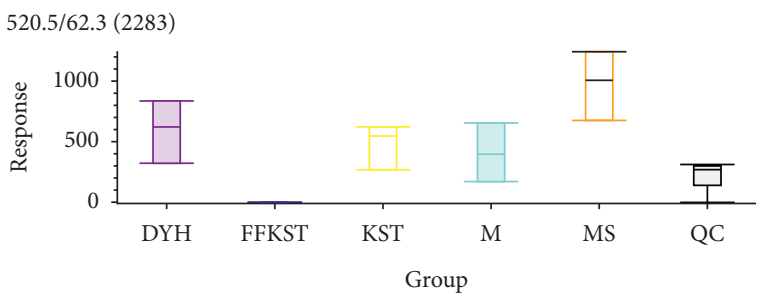

(h)

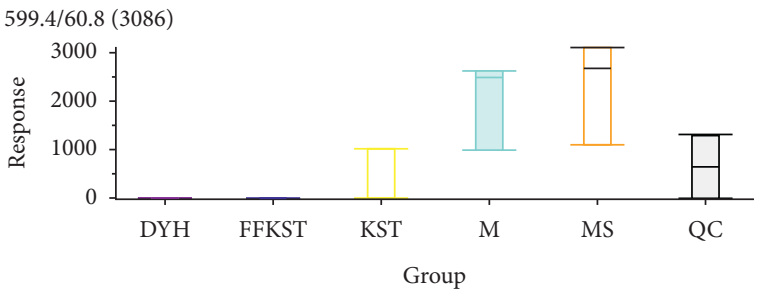

(j)

Group

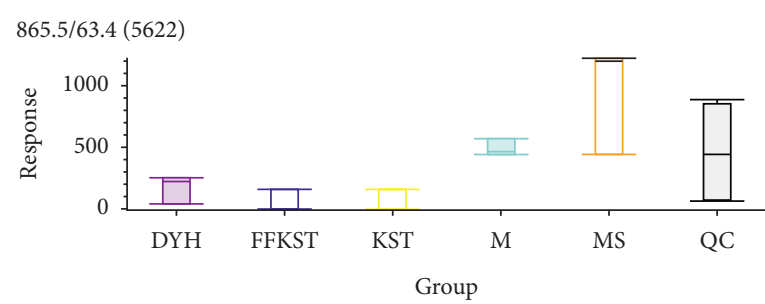

(k)

FIGURE 10: The response signals of 11 differential compounds in FFKST compared to $M$ in each group $(P<0.01)$ in the positive ion mode (results were output by the software MarkView 1.3). 


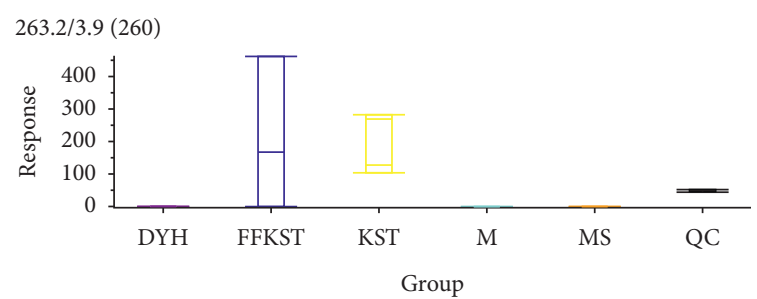

(a)

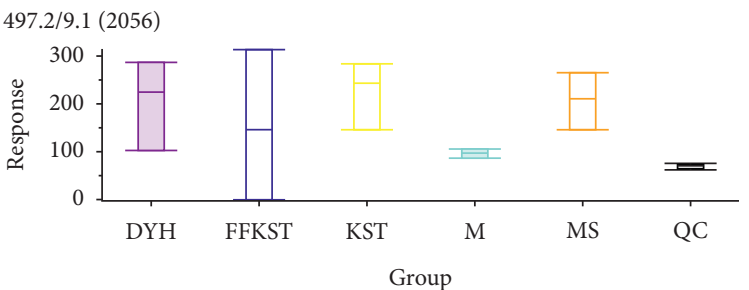

(c)

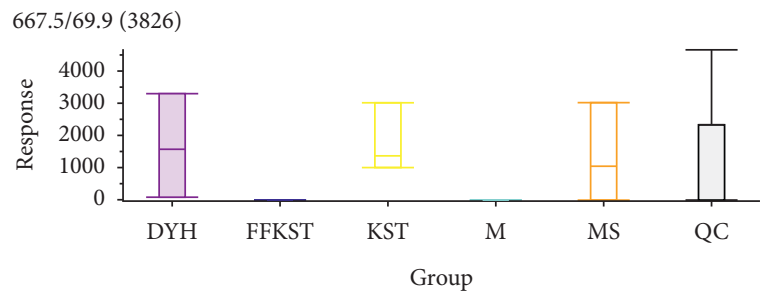

(e)

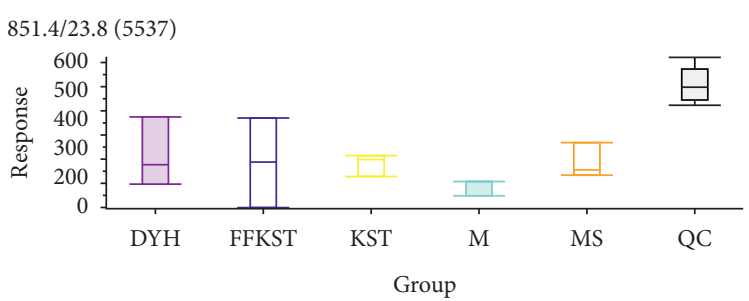

(b)

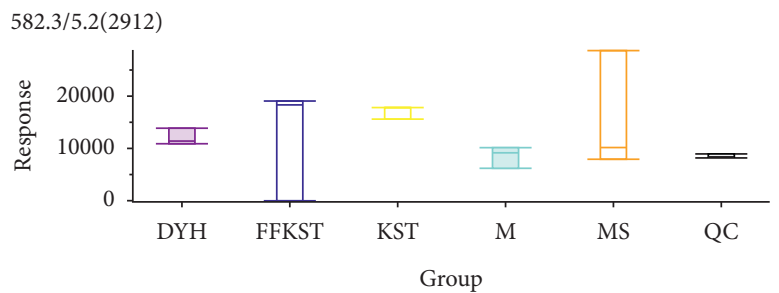

(d)

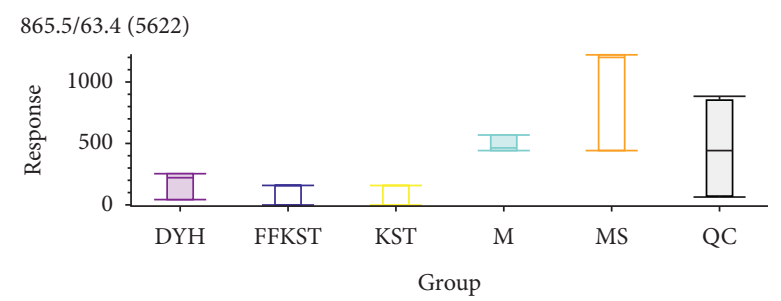

(f)

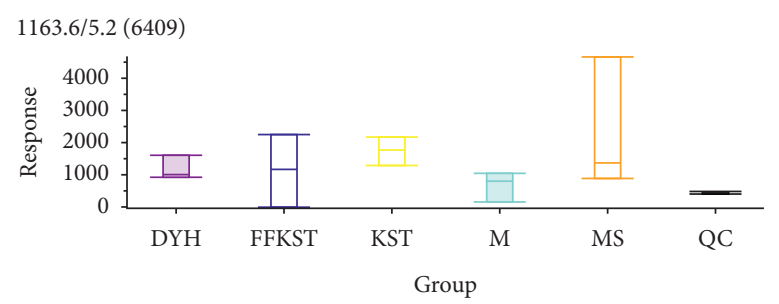

(g)

FIgURE 11: The response signals of 71 differential compounds in KST compared to $\mathrm{M}$ in each group $(P<0.01)$ in the positive ion mode (results were output by the software MarkView 1.3).

FFKST compared to $\mathrm{M}$, and 18 differential compounds were identified in KST compared to M. In the positive ion mode, 11 and 7 differential compounds were identified in FFKST and KST compared to the model group (M), respectively. Due to the large difference in the related substances produced by the action of the indirubin group (DYH) and the lack of related intersection of different products, the specifically different substances in DYH were not listed in this study. From the results of the analysis, the main discovery under the negative ion was the change in fatty acids and bile acids. In our previous study, we found that the metabolism of various unsaturated fatty acids, such as arachidonic acid and docosatrienoic acid, was abnormal in UC rats. Compound Sophora decoction could significantly regulate the metabolism of those compounds in UC mice and sufficiently reduce the inflammatory response and promote the repair of ulcer foci, which also fully demonstrated the integrated regulatory effect of traditional Chinese medicine. Matrine and oxymatrine possess antioxidant activity, and they can regulate blood lipids and protect liver cells [18], which could also inflict changes in fatty acids and bile acids in the body.

The main discovery in the positive ion mode was the change in the small peptides. Because a small peptide is originated from different proteins and it is not clear at this stage which proteins are the breakdown products, it is less significant in biological perspectives. In contrast, the changes in the fatty acids and bile acids in the negative ion mode were more consistent with our expectations. Figures 8 and 9 show the response signals of the 26 and 18 differential compounds in each group in negative ion mode, respectively $(P<0.01)$. However, Figures 10 and 11 show the response signal of the 11 and 7 differential compounds in each group in the positive ion mode, respectively $(P<0.01)$.

\section{Discussion and Conclusion}

The intestinal inflammatory damage and the pathological process of UC are closely related to the oxidative stress. 
Excessive oxygen radicals are involved in different defense mechanisms, including sterilization. Also, it often causes damage to healthy tissues. When the antioxidant system weakens or oxygen radicals are overproduced, oxygen-free radicals can cause damage to surrounding tissues, denature the protein molecules, inactivate enzymes, and even cause cell-death by promoting inflammatory exudation and edema. Excessive free radicals in the intestinal mucosa of UC patients could covalently combine with the multichain unsaturated fatty acids on the cell membrane, triggering a chain reaction of lipid peroxidation $[19,20]$. Matrine and oxymatrine can ameliorate the oxidative stress in cells by reducing the absorption of the free fatty acids, enhancing the oxidative utilization of the free fatty acids, enhancing the activity of antioxidant enzymes in cells, and reducing lipid peroxidation $[21,22]$. Due to the complex pathogenesis of ulcerative colitis, although the inhibition of excessive antioxidant stress response could provide some limited relief, it cannot produce a significant therapeutic effect.

Compound Sophora decoction effectively treats UC and prevents its recurrence. Modern pharmacological studies have shown that Radix Sophora flavescens decoction has antibacterial, anti-inflammatory, and leukocyte-increasing effects. Sanguisorba decoction could significantly shorten the bleeding and coagulation time, decrease the permeability of capillaries, reduce exudation, minimize tissue edema, form a protective film on the wound surface, promote the healing of the wound, and exert an antibacterial effect. Indigo naturalis decoction has a broad-spectrum antibacterial effect. Bletilla striata decoction contains colloid components, which could significantly shorten the bleeding and coagulation time, protect gastrointestinal mucosa, promote the formation of granulation tissue, and also exert an antibacterial effect. Therefore, each medicinal component of the compound Sophora decoction can synthetically coordinate the function. It can heal the wound and ulcer of the intestinal mucosa, repair the intestinal mucosal barrier, reduce the inflammatory response of the intestinal tract, and improve the immune function of the body. Many investigations have confirmed that the compound Sophora decoction can improve the immune function in $\mathrm{UC}$ and reduce colonic mucosal IkB-a and NF-kB expression, thereby inhibiting TNF-a and IL-6 expressions. Simultaneously, by inhibiting the activity of STAT 6 and disrupting the balance of Th1/Th2 cells, it could improve the inflammatory state in UC and achieve a better therapeutic effect against UC [23-25]. Unlike the monomers, the compound acts through the multipathway and multitarget mechanism. This hypothesis was also confirmed by the difference analysis results of this experiment. By comparing the differences of the serum principal components in the administered groups, we found that the FFKST group could exhibit enhanced synthesis of the serum principal components. However, the mixture of matrine and oxymatrine monomers did not cause the same changes in the serum principal components at the same dose.

In this study, the serum principal components of the target group were compared to the model group for detecting the substances with significant differences and accurately identify the difference in the serum principal components with or without drug intervention. Figures 8-11 show the differences in the response signals of each substance from each group in Tables 1-4. We checked horizontally at the vertical positions of each group; an intersection denotes no significant difference between the groups. Significant differences among the groups are indicated by the absence of any intersection with complete staggering at the vertical level. In the negative ion mode, 26 different compounds were detected in the compound Sophora decoction group (FFKST) compared to the model group (M), and five of them were solely present in FFKST with the retention times of 2.0, 3.4, 4.9, 6.6, and 7.6, which were metabolic intermediates. The specific metabolic pathways and pharmacochemical actions should be further studied. Five compounds from the 26 with retention times of 2.7, 43.0, 50.1, 56.2, and 59.4 can also be detected in KST, which were endogenous binders. However, the content difference was significant $(P<0.01)$. Although the type of substance was the same, different contents of the same substances could produce different therapeutic effects. The above results are consistent with the results of matrine and oxymatrine group (KST) in the negative ion mode. In the model group, the components with the retention times of 18.2 and 29.7 were identified as gingerol and glycolic acid. They have anti-inflammatory and analgesic activity. In the absence of drug intervention, the body produces specific immunity, stimulates the synthesis of endogenous active substances, and fights inflammation. However, an organism cannot self-heal when the immunity is low. Indirubin promotes the expression of compact junction proteins, regulates the permeability of intestinal epithelial cells, and reduces the passage of bacteria, endotoxins, and macromolecules through the mucosal barrier into the submucosa. Water molecules and ions pass through the hole where the cord is joined. A charge selective barrier between the intestinal cavity and intestinal mucosa can affect the colonic epithelial cell proliferation/apoptosis and cell migration/ repair and regulate the local immune disorders to achieve the therapeutic effect against UC [26, 27]. Although the components in DYH were not researched thoroughly due to the lack of correlation in different principal component substances with other groups, Figures 8-11 can still provide information about the corresponding components in $\mathrm{DYH}$, giving a feedback of differences in both quality and quantity.

In the positive ion mode, most of the 11 differential compounds were small peptides (product of endogenous protein decomposition), which were identified in the model group but were absent in the FFKST group. This study directs further exploration of the pathological mechanism of small peptides. Most of the 7 differential compounds characterized in the comparison between the KST and $M$ groups exhibited signal responses in FFKST and MS, once again proving that the traditional Chinese medicine compound is more comprehensive than monomers. Noteworthy, the differential compounds, we studied, were both endogenous and exogenous. It does not contain the exogenous ingredients, such as matrine and 
oxymatrine or indirubin, which were administered in the KST and DYH but not in M (which may produce a significant difference in expectation). These compounds could be detected in the blood in the positive ion mode; however, their content was very low. According to the software screening results, the difference compounds were the first 8000 substances with a $P$ value ranging from small to large $(P<0.01$ or $P<0.05)$. As content of these compounds was very low and negligible, they were not included in the results.

\section{Data Availability}

All data, models, or code generated or used during the study are available from the corresponding author by request.

\section{Conflicts of Interest}

The authors declare that there are no conflicts of interest regarding the publication of this paper.

\section{Authors' Contributions}

Wanjin Sun and Junjie Zhang contributed equally to this work. Junjie Zhang is the co-first author.

\section{References}

[1] D. Zuo, X. Liu, Z. Shou et al., "Study on the interactions between transplanted bone marrow-derived mesenchymal stem cells and regulatory $\mathrm{T}$ cells for the treatment of experimental colitis," International Journal of Molecular Medicine, vol. 32, no. 6, pp. 1337-1344, 2013.

[2] X. M. Zhang, Z. X. Shou, and H. Fan, "Effect of QRZSLXF on colon tissue repair in rats with ulcerative colitis," Journal of Traditional Chinese Medicine, vol. 55, no. 20, pp. 1764-1767, 2014.

[3] D. Zuo, Q. Tang, H. Fan et al., "Modulation of nuclear factor$\kappa \mathrm{B}$-mediated pro-inflammatory response is associated with exogenous administration of bone marrow-derived mesenchymal stem cells for treatment of experimental colitis," Molecular Medicine Reports, vol. 11, no. 4, pp. 2741-2748, 2015.

[4] P. Q. Zhou, H. Fan, H. Hu et al., "Role of DOR-B-arrestin1$\mathrm{Bcl} 2$ signal transduction pathway and intervention effects of oxymatrine in ulcerative colitis," Huazhong University of Science and Technology, vol. 34, no. 6, p. 1672, 2014.

[5] S.-T. Wu, Study on the Effective Components and Mechanism of Compound Sophorae Decoction against Ulcerative Colitis, Hubei University of Chinese Medicine, Wuhan, China, 2017.

[6] A. Schrattenholz, K. Groebe, and V. Soskic, "Systems biology approaches and tools for analysis of interactomes and multitarget drugs," Methods in Molecular Biology, vol. 662, pp. 29-58, 2010.

[7] Z. Yi, H. Fan, X. Liu, Q. Tang, D. Zuo, and J. Yang, "Adrenomedullin improves intestinal epithelial barrier function by downregulating myosin light chain phosphorylation in ulcerative colitis rats," Molecular Medicine Reports, vol. 12, no. 3, pp. 3615-3620, 2015.

[8] X. L. Wang, Z. C. Hong, and S. T. Wu, "Determination of matrine and oxymatrine in compound sophora decoction by
HPLC," Central South Pharmacy, vol. 15, no. 10, pp. 14261429, 2017.

[9] C. Z. Zhou, N. Jiang, and C. H. Zhou, "Study on the intervention and mechanism of compound sophora decoction in rats with ulcerative colitis model," China Pharmacist, vol. 19, no. 10, pp. 1816-1820, 2016.

[10] Z. C. Hong, X. Y. Duan, Q. Caia et al., "Study of compound sophorae decoction in the treatment of ulcerative colitis by tissue extract metabonomics approach," Journal of TCM, vol. 15, p. 40, 2020.

[11] H. Fan, Y. Liao, Q. Tang et al., "Role of $\beta_{2}$-adrenoceptor$\beta$-arrestin2-nuclear factor- $\kappa \mathrm{B}$ signal transduction pathway and intervention effects of oxymatrine in ulcerative colitis," Chinese Journal of Integrative Medicine, vol. 18, no. 7, pp. 514-521, 2012.

[12] H. Fan, X.-X. Liu, L.-J. Zhang et al., "Intervention effects of QRZSLXF, a Chinese medicinal herb recipe, on the DOR$\beta$-arrestin1-Bcl2 signal transduction pathway in a rat model of ulcerative colitis," Journal of Ethnopharmacology, vol. 154, no. 1, pp. 88-97, 2014.

[13] X.-Y. He, Q.-C. Liu, W. Peng, Y.-L. Huang, and C.-J. Wu, "Bioactivities and serum pharmacochemistry ofQi-Wei-XiaoYan-Tang," Pharmaceutical Biology, vol. 51, no. 5, pp. 629-634, 2013.

[14] G. T. Zhu, S. C. Wang, Z. J. Huang et al., "Rewiring of the fruit metabolome in tomato breeding," Cell, vol. 172, no. 1-2, pp. 249-261, 2018.

[15] Z. He, Y. Wang, Y. Zhang, H. Cheng, and X. Liu, "Stereoselective bioaccumulation of chiral PCB 91 in earthworm and its metabolomic and lipidomic responses," Environmental Pollution, vol. 238, pp. 421-430, 2018.

[16] J. K. Zhang, P. Wang, X. Wei et al., "A metabolomics approach for authentication of ophiocordyceps sinensis by liquid chromatography coupled with quadrupole time-of-flight mass spectrometry," Food Research International, vol. 76, pp. 489-497, 2015.

[17] J. K. Zhang, Q. H. Yu, H. Y. Cheng et al., "Metabolomic approach for the authentication of berry fruit juice by liquid chromatography quadrupole time-of-fight mass spectrometry coupled to chemometrics," Journal of Agricultural \& Food Chemistry, vol. 66, no. 30, pp. 8199-8208, 2018.

[18] L. H. Huang, Y. M. Zhong, X. H. Xiong et al., "The disposition of oxymatrine in the vascularly perfused rat intestine-liver preparation and its metabolism in rat liver microsomes," Journal of Pharmaceutical Sciences, vol. 105, no. 2, pp. 897903, 2016.

[19] S. Deng, Q. Tang, X. Duan et al., "Uncovering the anticancer mechanism of compound sophorae decoction against ulcerative colitis-related colorectal cancer in mice," Evidence-Based Complementary and Alternative Medicine, vol. 2019, Article ID 8128170, 16 pages, 2019.

[20] M. Xu, X.-Y. Duan, Q.-Y. Chen et al., "Effect of compound sophorae decoction on dextran sodium sulfate (DSS)-induced colitis in mice by regulating Th17/Treg cell balance," Biomedicine \& Pharmacotherapy, vol. 109, pp. 2396-2408, 2019.

[21] Z. Zou, D. Zuo, J. Yang, and H. Fan, "The ANXA1 released from intestinal epithelial cells alleviate DSS-induced colitis by improving NKG2A expression of natural killer cells," Biochemical and Biophysical Research Communications, vol. 478, no. 1, pp. 213-220, 2016.

[22] W Liu, S. Baker, R. Baker, and L. Ziu, "Antioxidant mechanisms in nonalcoholic fatty liver disease," Current Drug Targets, vol. 16, no. 12, pp. 1301-1314, 2015. 
[23] D. E. ZhuBaker and J. E. Friedman, "Developmental origins of nonalcoholic fatty liver disease," Pediatric Research, vol. 75, no. 1-2, pp. 140-147, 2014.

[24] P.-Q. Zhou, H. Fan, H. Hu et al., "Role of DOR-P-arrestin1$\mathrm{Bcl} 2$ signal transduction pathway and intervention effects of oxymatrine in ulcerative colitis," Journal of Huazhong University of Science and Technology [Medical Sciences], vol. 34, no. 6, pp. 815-820, 2014.

[25] Q. Chen, X. Duan, M. Xu et al., "BMSC-EVs regulate Th17 cell differentiation in UC via H3K27me3," Molecular Immunology, vol. 118, pp. 191-200, 2020.

[26] K. Inagaki-Ohara, F. N. Dewi, H. Hisaeda et al., "Intestinal intraepithelial lymphocytes sustain the epithelial barrier function against eimeria vermiformis infection," Infection and Immunity, vol. 74, no. 9, pp. 5292-5301, 2006.

[27] D. Gunzel, P. Florian, J. F. Richter et al., "Restitution of single cell defects in the mouse colon epithelium differs from that of cultured cells," American Journal of Physiology-Regulatory, Integrative and Comparative Physiology, vol. 290, no. 6, pp. 1496-1507, 2006. 